\title{
Remediation of learners struggling with communication skills: a systematic review
}

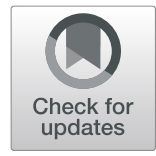

Deema Al-Sheikhly ${ }^{1 *}$, Linda Östlundh² and Thurayya Arayssi ${ }^{3}$

\begin{abstract}
Background: Communication skills is a core area of competency for healthcare practitioners. However, trainees deficient in those skills are not identified early enough to address the deficiency. Furthermore, faculty often struggle to identify effective remediation strategies for those who fail to meet expectations. We undertook a systematic review to determine which assessment methods are appropriate to identify learners that struggle with communication skills and the strategies used to remediate them.

Methods: The literature was searched from January 1998 through to May 2019 using academic databases and grey literature. Trainees were defined as healthcare practitioners in undergraduate, graduate and continuing education. Characteristics of studies, assessment and intervention strategies and outcomes were synthesized qualitatively and summarized in tables.
\end{abstract}

Results: From an initial 1636 records, 16 (1\%) studies met the review criteria. Majority of the learners were medical students. A few studies (44\%) included students from other disciplines, residents and physicians in practice. The remediation programs, in the studies, ranged from 1 week to 1 year. Around half of the studies focused solely on learners struggling with communication skills. The majority of studies used a format of a clinical OSCE to identify struggling learners. None of the studies had a single intervention strategy with the majority including an experiential component with feedback.

Conclusions: A few studies collectively described the diagnosis, remediation intervention and the assessment of the outcomes of remediation of communication skills. For a remediation strategy to be successful it is important to ensure: (i) early identification and diagnosis, (ii) the development of an individualized plan and (iii) providing reassessment with feedback to the learner.

Keywords: Assessment, Communication skills, Remediation, Intervention, Systematic review, Healthcare practitioners, Learners

\section{Background}

Interpersonal and communication skills are an integral element of quality patient care and are recognized as a core area of competency for medical students, residents, and practicing physicians [1-5]. Furthermore, effective communication and empathic relationships with healthcare practitioners are highly valued by patients and their families [6-10] with compromised clinical care and an overall

\footnotetext{
* Correspondence: dea2006@qatar-med.cornell.edu

${ }^{1}$ Division of Continuing Professional Development, Weill Cornell Medicine Qatar, Doha, Qatar

Full list of author information is available at the end of the article
}

lower satisfaction with care being associated with poor communication [11-13]. Hence formal training and assessment programs at the undergraduate, graduate and continuing education levels are needed [14-17]. Examples of these include Objective Structured Clinical Examination (OSCE) with a Standardized Patient (SP), that assesses clinical skills in a standardized setting. Although medical schools have a variety of methods to teach communication skills, there still remains a considerable gap. Not all communication curricula are based on a specific validated framework, nor incorporate a patient-centered communication approach, nor foster professional and personal

C C The Author(s). 2020 Open Access This article is licensed under a Creative Commons Attribution 4.0 International License, which permits use, sharing, adaptation, distribution and reproduction in any medium or format, as long as you give appropriate credit to the original author(s) and the source, provide a link to the Creative Commons licence, and indicate if changes were made. The images or other third party material in this article are included in the article's Creative Commons licence, unless indicated otherwise in a credit line to the material. If material is not included in the article's Creative Commons licence and your intended use is not permitted by statutory regulation or exceeds the permitted use, you will need to obtain permission directly from the copyright holder. To view a copy of this licence, visit http://creativecommons.org/licenses/by/4.0/ The Creative Commons Public Domain Dedication waiver (http://creativecommons.org/publicdomain/zero/1.0/) applies to the data made available in this article, unless otherwise stated in a credit line to the data. 
growth. Additionally, the learners' communication skills may not always be assessed directly and the quality of the program may not be evaluated [17].

In 1999, the Accreditation Council for Graduate Medical Education (ACGME) and the American Board of Medical Specialties (ABMS) stated that "interpersonal and communication skills that result in effective information exchange and partnering with patients, their families, and professional associates" is a core area of competency for residents and practicing physicians [1, 4]. Additionally in 2004, the National Board of Medical Examiners (NBME), the Federation of State Medical Boards (FSMB), and the Educational Commission for Foreign Medical Graduates (ECFMG) implemented the Step 2 Clinical Skills (CS) Examination [18]. One of three subcomponents of the exam is Communication and Interpersonal Skills, which requires medical students or graduates to "establish rapport with the patients, gather and provide information, help the patient make decisions and provide counseling when appropriate and in a professional manner" [18].

Despite the importance of communication skills to the training of future healthcare practitioners and the requirement to demonstrate competence in those skills at all levels of the medical continuum, faculty and residency program directors often struggle with identifying effective remediation strategies for those who fail to meet expectations [19-21]. This has mainly been due to the fact that remediation is a time consuming process that can be daunting and cumbersome [22] and that remediation of non-cognitive problems is more challenging than remediation of cognitive problems [20,23].

The literature has shown that policies and guidelines for best practice are needed to improve the quality of the remediation process and to increase the confidence of educators in applying specific remediation strategies according to the learner's skill deficit in all areas of competencies [23, 24]. A variety of remediation strategies have been utilized with most consisting of three steps: identification/diagnosis, remediation intervention, and re-assessment [25-27]. Hauer et al. proposed a four-step model which included: (i) initial assessment to identify deficiencies using multiple assessment tools, (ii) diagnosis and development of an individualized learning plan, (iii) deliberate practice, feedback, and reflection, and (iv) reassessment [23]. A structured seven-step approach of relationship-centered care, coaching and effective feedback was also found to be an effective model to successfully remediate learners in communication and interpersonal skills [28]. Some of the key steps included establishing a supportive learning environment, listening to the learner, encouraging reflective practice, developing a learning plan and documenting progress.

The literature on challenges in identifying and remediating learners struggling with communication skills are wide and varied [28]. Therefore, the goal of this systematic review was to determine the appropriate assessment tools used to identify learners struggling in communication skills, the strategies used to remediate them and to discuss the best practice recommendations proposed by the authors. In this study we defined remediation as "additional teaching above and beyond the standard curriculum, individualized to the learner who without the additional teaching would not achieve the necessary skills for the profession" [29].

To achieve this, our research question was:

How do you diagnose a trainee struggling with communication skills and what are the effective remediation strategies?

\section{Method}

In this systematic review a PRISMA (Preferred Reporting Items for Systematic Reviews and Meta-Analysis) flow diagram was utilized for reporting the study selection.

\section{Data sources and search strategy}

A comprehensive, search for literature was performed in the academic databases PubMed, MEDLINE (OVID), EMBASE (OVID), CINAHL (EBSCO), PsycInfo (OVID), Web of Science and Scopus and in sources of grey literature. Pre-searches to identify relevant search strategies, search terms and information sources were conducted in March-June, 2018, and the final search was carried out in June 2018. An update of the search in PubMed and Scopus were performed in May 2019 to ensure inclusion of the latest published studies on remediation in communication for healthcare practitioners before completing the manuscript.

PubMed was used to systematically develop a search string, which later was applied in the other databases. All selected keywords were searched both in the fields "Abstract" and "Article Title" (alternatively "Topic") and in MeSH/Subject Headings/Thesaurus when available. No filters or limitations were applied to retrieve the largest number of result and to avoid excluding pre-indexed materials. Language, document type, and publication year restrictions were instead included in the exclusion criteria for the screening process. We defined trainees as healthcare practitioners in undergraduate, graduate and continuing education. For the purpose of this study we defined healthcare practitioners as individuals who may be involved in healthcare delivery (for example: physicians, nurses, dentists, physiotherapists and pharmacists). A full search log, including detailed search strings for all included information sources, results and notes is available in Appendix.

Searches for grey literature were conducted in ProQuest Dissertation and Thesis, Ethos, Open Grey and BASE, The New York Academy of Grey Literature Reports and in the library catalogues for British Library, Library of congress and WorldCat. Due to lack of advanced search features in many of the grey resources, broader search strings than the 
one used in the academic databases had to be applied. The grey search was updated in May 2019. A full search log can be found in Appendix.

All the references were uploaded into Covidence (Melbourne, Australia), systematic review software for blinded screening. Duplicate detection and removal were carried out using this software.

To complete the selection of relevant references for the review, a systematic hand screening of references lists in studies identified to be included in the systematic review was also carried out. Two additional studies were identified eligible for the systematic review.

\section{Study selection and title and abstract review}

Articles were included if they were original research on remediation in the area of interpersonal and communication skills. Articles that were not written in the English language, systematic reviews, conference abstracts, proceedings, book chapters, comments, editorials or letters and publications prior to 1998 were excluded. We wanted to limit the review to primary studies following the implementation of the Accreditation Council for Graduate Medical Education's
(ACGME) outcome project where competencies for training, including communication skills, were defined and implemented. The search in academic databases and in grey sources yielded 1636 articles (Fig. 1). Based on the title and abstract, the two reviewers (DA and TA) screened the articles using Covidence and excluded articles that were clearly irrelevant. The screening in Covidence was blinded. In situations where it was difficult to determine eligibility based on the title and abstract review the article was included for full article review. The authors met regularly and all uncertainties were resolved by consensus. Only articles that described an assessment tool to identify struggling learners as well as an intervention methodology or remediation strategy were included. Articles with an assessment and remediation strategy but no clear outcome were also included.

\section{Data extraction}

Data were entered into a structured extraction framework that we created. The framework included information on the article (Year, participant level, participant number, country of study), assessment tool, remediation strategy and intervention outcome. One author (DA) extracted the data.

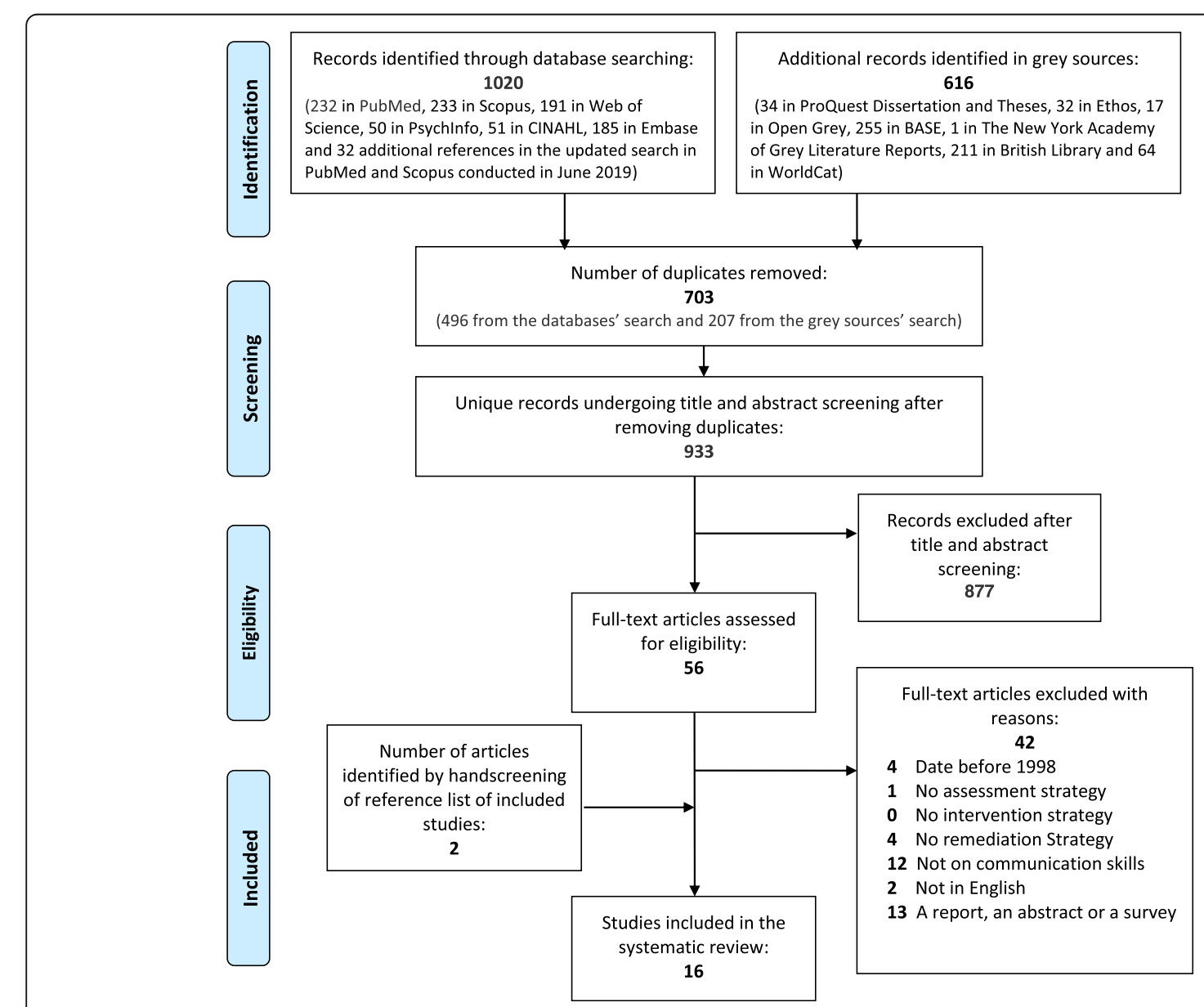

Fig. 1 PRISMA flow diagram [30] 
The Medical Education Research Study Quality Instrument (MERSQI) used to evaluate the methodological quality of experimental, quasi-experimental, and observational studies in medical education was used [31]. The tool includes 10 items, reflecting 6 domains of study quality [study design, sampling, type of data (subjective or objective), validity, data analysis, and outcomes]. The potential range of a MERSQI score is 5-18. Each study was scored at the highest possible level.

We used the Kirkpatrick's four-level model for evaluation of educational interventions to classify the outcomes of the studies that met our selection criteria [32]:

- Level 1: Participant reaction assessed (learner satisfaction).

- Level 2: Participant learning assessed (changes in knowledge and skills).

- Level 3: Participant behavioral change assessed (application in practice).

- Level 4: Results (changes in institutional practice and patient outcome).

\section{Data synthesis}

Characteristics of studies, assessment and intervention strategies and outcomes were synthesized qualitatively and summarized in tables. Articles that described similar assessment tools or similar interventions were grouped to facilitate the analysis. We used the consensus mean MERSQI scores with standard deviations to describe the overall quality of included studies. Meta-analysis was not possible, given the heterogeneity of assessment tools, interventions and outcome measurements.

\section{Results}

\section{Characteristics of eligible studies}

From the 1636 records found, 1020 were identified through electronic database searching and 616 were identified through other resources such as databases for grey literature and by systematically reviewing citations in studies selected to be included in the review. After de-duplication, a total of 933 references were identified for a first review. The total number of articles that were eligible for inclusion through review of titles, abstracts and full texts was 16 (Fig. 1). Table 1 summarizes information on the country of the study, the level of struggling learner, number of remediated learners, assessment tools, interventions and outcomes. Struggling learners in the studies were predominantly students $(n=11$, $69 \%$ ), followed by residents ( $n=4,25 \%)$, and one study included family physicians and specialists $(n=1,6 \%)$. Only one study included pharmacy students (6\%), 14 included learners in medicine (88\%) and one included both nursing and medical students (6\%). Eight studies were conducted in the US, three in the UK, two in Canada, one in Belgium, one in Australia and one in Korea. Only seven studies focused solely on learners struggling with communication skills [33-39] while the remaining studies addressed multiple deficiencies.

\section{Quality of studies}

The mean consensus MERSQI score was 10.5 (range 5.511), with a standard deviation of 1.67 and a median score of 8.5, indicating that the overall study quality was not high. Total consensus MERSQI scores for each paper are shown in Table 2. Mean domain scores were highest for type of data (2.63), data analysis (2.19) and sampling (2.06); they were lowest for validity evidence (1.17) and study design (1.13). Most of the studies (81.3\%) were single group crosssectional or single group post-test only. One study was a retrospective review of records [40], two studies were surveys of medical schools [24, 39] and one study was a survey of surgery residency programs [20].

\section{Assessment methods used to diagnose struggling trainees}

Table 1 provides details of the assessment tools that were used in the studies to diagnose trainees struggling with communication skills and Table 3 provides a summary of the overall assessment methods used in the studies reviewed. Most studies $(n=10,62.5 \%)$ used a format of a clinical OSCE, a tool to assess clinical skills in a controlled setting, to identify struggling learners [33-35, $37-39,41-44]$, four (25\%) used a 360-degree or peer evaluation tool $[42,45-47]$, one study did not address assessment methods used [21] and another identified struggling learners by their failure to meet criteria in one or more CanMed Roles but did not expand on what tools were used to achieve this [40].

(Table 3: Summary of Assessment Methods and Remediation Strategies) - Insert near here.

\section{Remediation interventions and outcomes}

The studies included a wide range of intervention strategies such as one-on-one coaching/mentoring, tutorials, individual and group work, focus reading, SP exercises, role-play, videotape review, and counseling (Table 1). None of the studies had a single intervention strategy with the majority including an experiential component with feedback. Half of the studies $(n=8)$ developed a remediation course or program [33-36, 38, 41, 43, 47] with the duration of the intervention ranging from as short as a weeklong course [34] to a longitudinal oneyear program [35].

Five studies (35.3\%) had a program director or a committee to devise an individualized remediation plan that included input from learners [20, 37, 40, 45, 46] and one of those studies used the CanMed Roles as a framework for the development of the plan [40]. In one Canadian study on improving physicians in practice a variety of intervention strategies were used. 
Table 1 Summary of characteristics of eligible studies

\begin{tabular}{|c|c|c|c|c|c|}
\hline $\begin{array}{l}\text { First Author } \\
\text { and year }\end{array}$ & $\begin{array}{l}\text { Number \& } \\
\text { Struggling } \\
\text { learner } \\
\text { Characteristic } \\
\text { \& Country }\end{array}$ & $\begin{array}{l}\text { MERSQI } \\
\text { Score }\end{array}$ & $\begin{array}{l}\text { Description of Assessment for } \\
\text { identifying struggling learner }\end{array}$ & Intervention & $\begin{array}{l}\text { Outcomes (Kirkpatrick level of } \\
\text { evaluation) }\end{array}$ \\
\hline $\begin{array}{l}\text { Bodenberg } \\
2015^{(44)}\end{array}$ & $\begin{array}{l}8 \\
\text { Pharmacy } \\
\text { Student } \\
\text { US }\end{array}$ & 8 & $\begin{array}{l}\text { - Midpoint evaluation and concern } \\
\text { cards to alert the Director of } \\
\text { Experiential Education of } \\
\text { potential behavioral or learning } \\
\text { issues. }\end{array}$ & $\begin{array}{l}\text { The Director of Experiential } \\
\text { Education creates a performance } \\
\text { improvement plan. } \\
\text { This individualized plan takes into } \\
\text { account input from the student, }\end{array}$ & $\begin{array}{l}\text { The on-time graduation using the } \\
\text { developed remediation plan was } \\
\text { seven of eight students ( } 88 \% \text { ), and } \\
\text { the overall graduation success rate } \\
\text { was eight of eight students }\end{array}$ \\
\hline
\end{tabular}

- The faculty developed a section preceptor, and the Director of

in the student evaluation form in Experiential Education.

(100\%).

which the preceptor can suggest A list of communication tools

longitudinal monitoring or includes:

remediation needed for the $\quad$ Direct observations of the

student.

- $9 \%$ had a communications skills deficiency.

student during case

presentations,

- Counseling,

- Drug information,

- Topic presentations, and

- An oral "End of Block" exam.

$\begin{array}{ll}\text { Chang } 2008 & 23 \\ & \text { Medical } \\ & \text { Student } \\ & \text { US }\end{array}$

5.5 The clinical performance examination (CPX) is an eightstation, high-stakes examination in which SPs assess early Year 4 medical students' competence in clinical and communication skills. Students scoring $\geq 2$ standard deviations (SD) below the class average in one or both skill domains (history taking and physical examination [H \& P] or patient-doctor interaction [PDI]) are required to remediate.
Developed a four-step CPX remedial training program that incorporates diagnosis of learner

problems, individual reflection, faculty feedback and supervised practice.

$\underline{\text { Step } 1}$ consists of an individual review in which:

- Students watch their own videotaped examinations,

- Reflect on their performances, and

- Develop personal learning goals. Step 2, the faculty remediation director:

- Watches the videotape independently and

- Generates a 'learning prescription',

Step 3 involves a one-to-one video review guided by the learning prescription and the student's self-identified goals with a designated faculty member.

In Step 4, students attend one or both evening workshops in $\mathrm{H}$ \& $\mathrm{P}$ or PDI, depending on their area of need.

Workshops consist of: an introductory 30-min didactic session addressing basic concepts; case-based skills exercises in which subsets of 2-3 students rotate between examination rooms with a faculty facilitator and an SP, and a whole-group concluding session to review learning goals.

Three different remediation strategies depending on year level:

- During the 2nd year of the

Bachelor degree, remedial teaching consists of one up to three extra training sessions in a small group.

- During the 1st year of the Master education, every student who failed is invited to exercise during
Students who require remedial training in clinical and communication skills appreciate individualized feedback and skills sessions with preceptors and SPs. According to personal communication, all students in the program succeeded.

\section{(Level 1)}

No outcome stated communication curriculum, using an OSCE with SP with specific objectives.

- Two examiners rate the student and come to a final mark after discussion. 
Table 1 Summary of characteristics of eligible studies (Continued)

\begin{tabular}{lllll}
\hline First Author & $\begin{array}{l}\text { Number \& } \\
\text { and year }\end{array}$ & MERSQI & Description of Assessment for & Intervention \\
& learner & Score & identifying struggling learner & Outcomes (Kirkpatrick level of \\
& Characteristic & & & evaluation) \\
& \& Country & & \\
\hline
\end{tabular}

\begin{tabular}{|c|c|c|c|}
\hline $\begin{array}{l}\text { Dowell } \\
2006^{(33)}\end{array}$ & $\begin{array}{l}28 \\
\text { Medical } \\
\text { Student } \\
\text { UK }\end{array}$ & 8 & $\begin{array}{l}\text { - OSCE to assess communication } \\
\text { skills "Consultation skills" as a } \\
\text { screening tool. } \\
\text { - Three 4-min OSCE to assess as- } \\
\text { pects of communication skills. } \\
\text { - Scoring was done using the } \\
\text { SEGUE framework by trained } \\
\text { tutors. }\end{array}$ \\
\hline
\end{tabular}

$\begin{array}{ll}\text { Goulet } & 220 \\ 2005^{(41)} & \text { Family } \\ & \text { Physician } \\ & \text { and } \\ & 85 \\ & \text { Specialist } \\ \text { Consultant } \\ \text { Canada }\end{array}$

16

IM,

Diagn Rad,
$1 \mathrm{~h}$ with a simulated patient. The student can formulate his/her own learning objectives. The whole session is videotaped and the taped is reviewed by one of the trainers in order to give feedback to the student. The student can rehearse this three times.

- During the 2nd year of the Master degree, the student exercises with a simulated patient in the presence of one of the trainers and gets immediate feedback.

- Attend a week of additional training.

- Interactive teaching contained many opportunities to develop, videotape, review and practice both basic and more complex consultation skills.

- At the end, a separate fourstation OSCE was used to assess skills using another three fourminute consultation skills stations plus an additional ten-minute assessment that allowed students to complete a basic consultation.

- This was videotaped to enhance feedback and reliability.

To improve Physician practice, the Professional Inspection Committee (PIC) may recommend that:

1. The physician participates in specific CME activities.

2. The CMQ admin committee imposes a remedial retraining.

Interventions include:

- Clinical training programs,

- Tutorials,

- Focused readings, and

- Various courses or workshops developed and organized by the medical schools in Quebec or the

- $70 \%$ of the retraining activities led to attainment of the training objectives;

. $15 \%$ led to partly attained objectives,

- $13 \%$ failed to lead to attainment of objectives, and

- $2 \%$ involved missing data or withdrawal.

The $70 \%$ resumed their practices.

(Level 2) CMO in association with the Quebec College of Family Physicians.

Every assessment process is

conducted using a:

- Standard grid listing the criteria

of quality of care,

- Chart keeping, and

- Office practices.

Sometimes a more in-depth evaluation is conducted:

- An evaluation of specialists'

clinical performance by a peer.

- The structured oral interview

(SOI). Six objective structured

clinical examination (OSCE)

stations were added to the SOI.

(includes knowledge, Physical

exam, Doctor-patient

relationship)

- Monthly evaluation from Faculty, · Individualized Education Plans peers and students. (IEP) that includes a listing of

-360-degree evaluation, which

competencies, intervention
12/16 successfully graduated from the IEP program.

(Level 2) 
Table 1 Summary of characteristics of eligible studies (Continued)

\begin{tabular}{|c|c|c|c|c|}
\hline $\begin{array}{l}\text { First Author } \\
\text { and year }\end{array}$ & $\begin{array}{l}\text { Number \& } \\
\text { Struggling } \\
\text { learner } \\
\text { Characteristic } \\
\text { \& Country }\end{array}$ & $\begin{array}{l}\text { MERSQI } \\
\text { Score }\end{array}$ & $\begin{array}{l}\text { Description of Assessment for } \\
\text { identifying struggling learner }\end{array}$ & Intervention \\
\hline & $\begin{array}{l}\text { Ob/Gyn, ER, } \\
\text { Gen Surg, } \\
\text { Peds } \\
\text { Residents } \\
\text { US }\end{array}$ & & $\begin{array}{l}\text { includes input from nurses and } \\
\text { case managers. } \\
\text { - Evaluations reviewed by Internal } \\
\text { Residency Review Committee } \\
\text { (IRRC). } \\
\text { - The IRRC charges the PD or Chief } \\
\text { Resident to counsel the resident } \\
\text { and monitor their progress. If no } \\
\text { improvement formal IEP may be } \\
\text { required. }\end{array}$ & $\begin{array}{l}\text { designed to address, } \\
\text { reassessment with objective } \\
\text { metrics and milestones for } \\
\text { completion of IEP. } \\
\text { - Resident input is solicited to } \\
\text { refine the IEP and to identify a } \\
\text { faculty mentor. } \\
\text { - Interventions include, remedial } \\
\text { tutorials, frequent meetings with } \\
\text { mentor, speech therapy, } \\
\text { counseling }\end{array}$ \\
\hline $\operatorname{Lin} 2001^{(34)}$ & $\begin{array}{l}1 \\
\text { Medical } \\
\text { Student } \\
\text { US }\end{array}$ & 8 & $\begin{array}{l}\text { - Clinical preceptors assessment } \\
\text { and end-of-year clinical practice } \\
\text { exam. } \\
\text { - OSCE/SP encounters in } \\
\text { communication Skills. }\end{array}$ & $\begin{array}{l}\text { A year-long intensive remedial cur- } \\
\text { riculum in communication skills. } \\
\text { Includes: } \\
\text { - Pairing with a clinical preceptor } \\
\text { for intensive skills training, } \\
\text { including a weekly preceptor } \\
\text { clinic, } \\
\text { - Structured readings, } \\
\text { - SP exercises, } \\
\text { - Communications workshops, and } \\
\text { - End-of-year standardized clinical } \\
\text { evaluations. }\end{array}$ \\
\hline
\end{tabular}

$\begin{array}{llll}\text { Malau-Aduli } & 18 & 11 & \begin{array}{l}\text { Students were identified for } \\ \text { 2013 }\end{array} \\ & \begin{array}{l}\text { Medical } \\ \text { Students }\end{array} & \begin{array}{l}\text { - Failure in examinations or } \\ \text { (4th and 5th } \\ \text { year) }\end{array} & \text { repeating the year. } \\ \text { Australia } & & \text { - Workplace-based assessments. }\end{array}$

$\begin{array}{ll}\text { Myung } & 23 \\ 2013^{(42)} & \text { Medical } \\ & \text { Students } \\ & \text { Korea }\end{array}$

Clinical performance examination (CPX) (8 stations with SP encounters using a checklist).

$\begin{array}{ll}\text { Rowland } & 225 \\ 2012^{(47)} & \text { Surgery } \\ & \text { Residents } \\ & \text { US }\end{array}$

9.5 - Identified by Directors: (Mock Oral Exams, Case Presentations, Journal Clubs, Mortality \& Morbidity conferences, outpatient clinics, and hospital and operating room settings).

- Failed Surgery Certifying Exam.

- Rowland Communication Skills Inventory used to identify candidates with severe
Outcomes (Kirkpatrick level of evaluation)
Remediation program developed based on socio-cognitive Self-

Efficacy beliefs to improve academic and clinical performance.

- A multi-dimensional ten-week support programbased on individual assessment offered primarily as a group learning experience.

- Individual counseling to provide psychological support.

The program consisted of the following elements:

- Presentation Skills workshops

- OSCE Practice (4-station) with

Clinical Teachers

- OSCE Practice with other

Students

- Bedside Teaching

Improvement in communication skills. Additionally the student unexpectedly wrote a 12-page guide to interview skills for his preclinical colleagues.

\section{(Level 2)}

Performance on all measures improved after the remediation program with statistically significant improvements on management plan (MP), diagnostic skills (DS), communication skills (CS) and number of stations passed (NSP).

All 18 participants in the remediation program were successful in their end of 4th year summative OSCE assessments. In their 5th year (eight of them) passed all their examinations without any support or intervention.

(Level 2)

A six-week remediation program (3 weeks Internal Medicine classes and 3 weeks Family Medicine classes). Includes:

- 1:1 tutoring sessions

- Re-examination

- Feedback from SP

Designed with 3 parts: Diagnosis, learning activities, and reexamination.

5-Day Oral Examination Course:

- Didactics,

- Mini oral examinations in suites,

- Individual assessments,

- Small-group exercises,

- Formal mock oral examinations,

- Individual debriefing sessions

with a general surgeon and a behavioral scientist that summarized individual
- Students' scores on the CPX

exam improved.

(Level 2)
Primary outcome measure is the successful completion of the Certifying Exam.

218 residents followed their remediation plan and successfully Passed the Surgery CE on first attempt

(Level 2) 
Table 1 Summary of characteristics of eligible studies (Continued)

\begin{tabular}{|c|c|c|c|c|c|}
\hline $\begin{array}{l}\text { First Author } \\
\text { and year }\end{array}$ & $\begin{array}{l}\text { Number \& } \\
\text { Struggling } \\
\text { learner } \\
\text { Characteristic } \\
\text { \& Country }\end{array}$ & $\begin{array}{l}\text { MERSQI } \\
\text { Score }\end{array}$ & $\begin{array}{l}\text { Description of Assessment for } \\
\text { identifying struggling learner }\end{array}$ & Intervention & $\begin{array}{l}\text { Outcomes (Kirkpatrick level of } \\
\text { evaluation) }\end{array}$ \\
\hline & & & $\begin{array}{l}\text { communication problems that } \\
\text { might need further assistance } \\
\text { with their communication skills } \\
\text { before entering the course. }\end{array}$ & $\begin{array}{l}\text { improvement, communication } \\
\text { competency, strengths and } \\
\text { weaknesses on the formal mock } \\
\text { oral examination, and a } \\
\text { remediation plan for future } \\
\text { improvement. Resident received } \\
\text { a personal digital video of their } \\
\text { formal mock oral for review and } \\
\text { self-critique and individual re- } \\
\text { mediation plan. } \\
\text { In 2007, course shortened to } 3 \\
\text { Days. }\end{array}$ & \\
\hline Ryan $2010^{(36)}$ & $\begin{array}{l}64 \\
\text { Nursing } \\
46 \\
\text { Medical } \\
\text { Students } \\
\text { UK }\end{array}$ & 7 & $\begin{array}{l}\text { A screening program in } \\
\text { communication and consultation } \\
\text { skills (CCS) using: } \\
\text { - Trained Standardized Patient } \\
\text { Educators (SPEs) } \\
\text { - A previously validated global } \\
\text { rating scale for CCS. } \\
\text { Almost three quarters of medical } \\
\text { students ( } 33 / 46 ; 72 \% \text { ) and } 81 \% \text { of } \\
\text { nursing students (56/64) passed } \\
\text { the CCS assessment in both } \\
\text { communication and attitudes } \\
\text { categories. }\end{array}$ & One-on-one CCS training. & (Level 2) \\
\hline $\begin{array}{l}\text { Saxena } \\
2009^{(24)}\end{array}$ & $\begin{array}{l}\text { Medical } \\
\text { Students } \\
\text { US }\end{array}$ & 11 & $\begin{array}{l}\text { Comprehensive Assessment Test } \\
\text { (Cross-disciplinary exam with SPs). } \\
\text { - Reviewing exam scores (96\%) } \\
\text { - Reviewing video of failing } \\
\text { student exam (57\%) } \\
\text { - Meeting with failing stud (49\%) }\end{array}$ & $\begin{array}{l}\text { - Precepted video review } \\
\text { - Preceptorship } \\
\text { - Independent Study (Web-Based } \\
\text { module, reading) } \\
\text { - Stud independently reviews } \\
\text { exam recording } \\
\text { - Practice with SP } \\
\text { - Skills workshops, seminars or } \\
\text { group discussions }\end{array}$ & $\begin{array}{l}\text { - Study measured confidence and } \\
\text { not outcomes of remediation. } \\
\text { (Level 2) }\end{array}$ \\
\hline $\begin{array}{l}\text { Sperry } \\
2010^{(37)}\end{array}$ & $\begin{array}{l}3 \\
\text { Medical } \\
\text { Students } \\
\text { (4th yr) } \\
\text { US }\end{array}$ & 11 & $\begin{array}{l}\text { Clinical performance examination } \\
\text { (CPX) using SP. } \\
\text { - Evaluation includes Medical } \\
\text { History, physical exam, } \\
\text { communication and relationship } \\
\text { issues, diagnosis and } \\
\text { management. }\end{array}$ & $\begin{array}{l}\text { Individualized Doctor-Patient Com- } \\
\text { munications and Psychosocial } \\
\text { Interviewing remediation curricu- } \\
\text { lum addresses communication } \\
\text { skills deficits: } \\
\text { - 2-week Didactic and experiential } \\
\text { components including role-play, } \\
\text { videos, personal reflections, per- } \\
\text { forming interviews, history taking } \\
\text { and Physical exam with patients } \\
\text { presenting to clinic. } \\
\text { - Observed Live and provided } \\
\text { feedback by family physicians. } \\
\text { - Written test assess knowledge of } \\
\text { communication strategies before } \\
\text { and after remediation. } \\
\text { - Patients completed a satisfaction } \\
\text { survey. }\end{array}$ & $\begin{array}{l}\text { - No difference in written test } \\
\text { scores. } \\
\text { - Patient satisfaction indicated } \\
\text { positive qualities with no } \\
\text { difference before and after. } \\
\text { - Preceptors' evaluation of students } \\
\text { indicated an improvement in CS. } \\
\text { - All passed CPX and one passed a } \\
\text { re-sit of USMLE-CS } \\
\text { (Level 2) }\end{array}$ \\
\hline $\begin{array}{l}\text { Torbeck } \\
2009^{(20)}\end{array}$ & $\begin{array}{l}\text { Surgery } \\
\text { Residents } \\
\text { US }\end{array}$ & 6 & $\begin{array}{l}\text { - Assessment tools used were not } \\
\text { addressed. }\end{array}$ & $\begin{array}{l}\text { Program director devise individual } \\
\text { remediation plans and monitor } \\
\text { progress. Most programs use } \\
\text { primarily } 3 \text { methods for } \\
\text { remediating residents: } \\
\text { - To increase direct observation of } \\
\text { the resident by the attending in } \\
\text { the clinic/operating room/wards, } \\
\text { - To have the resident undergo } \\
\text { psychological counseling, and }\end{array}$ & (Level 1) \\
\hline
\end{tabular}


Table 1 Summary of characteristics of eligible studies (Continued)

\begin{tabular}{|c|c|c|c|c|c|}
\hline $\begin{array}{l}\text { First Author } \\
\text { and year }\end{array}$ & $\begin{array}{l}\text { Number \& } \\
\text { Struggling } \\
\text { learner } \\
\text { Characteristic } \\
\text { \& Country }\end{array}$ & $\begin{array}{l}\text { MERSQI } \\
\text { Score }\end{array}$ & $\begin{array}{l}\text { Description of Assessment for } \\
\text { identifying struggling learner }\end{array}$ & Intervention & $\begin{array}{l}\text { Outcomes (Kirkpatrick level of } \\
\text { evaluation) }\end{array}$ \\
\hline & & & & $\begin{array}{l}\text { - To have the resident attend } \\
\text { organized professionalism or } \\
\text { communication workshops/ } \\
\text { seminars. } \\
\text { Among the other methods } \\
\text { reported: } \\
\text { - } 360^{\circ} \text { evaluations, } \\
\text { - Specific counseling with the PD, } \\
\text { - Reviewing How to Win Friends \& } \\
\text { Influence People on a weekly } \\
\text { basis with the program director, } \\
\text { - Have residents present } \\
\text { frequently, } \\
\text { - Sending a resident to an English } \\
\text { tutor, } \\
\text { - Recommending a speech/ } \\
\text { communication coach, and } \\
\text { - Counseling for problems related } \\
\text { to hostile relationships/ } \\
\text { interactions. }\end{array}$ & \\
\hline $\begin{array}{l}\text { Wiskin } \\
2013^{(38)}\end{array}$ & $\begin{array}{l}1 \\
\text { Medical } \\
\text { Student } \\
\text { UK }\end{array}$ & 8 & - Clinical OSCE & $\begin{array}{l}\text { - One-on-One coaching } \\
\text { - Individual and group teaching, } \\
\text { - Individual support and remedial } \\
\text { teaching, } \\
\text { - Workshop program, } \\
\text { - OSCE course. }\end{array}$ & No outcome stated \\
\hline $\begin{array}{l}\text { Zbieranowski } \\
2013^{(39)}\end{array}$ & $\begin{array}{l}100 \\
\text { Medical } \\
\text { Resident } \\
\text { Canada }\end{array}$ & 8 & $\begin{array}{l}\text { - Identified by Failure to meet } \\
\text { criteria of CanMEDS roles. } \\
\text { - Board of Examiners for } \\
\text { Postgraduate Programs (BOE-PG) } \\
\text { objectively review cases of } \\
\text { postgraduate students in } \\
\text { academic difficulty and } \\
\text { determine appropriate course of } \\
\text { action, which could include: } \\
\text { Remediation, probation, or } \\
\text { dismissal. } \\
\text { - } 49 \% \text { had weakness as } \\
\text { Communicator CanMed Role. }\end{array}$ & $\begin{array}{l}\text { CanMEDS Roles are used as the } \\
\text { organizational framework for the } \\
\text { individual formal remediation } \\
\text { plans developed by the residency } \\
\text { program director. }\end{array}$ & $\begin{array}{l}\text { 78\% Completed Residency } \\
\text { Education. } \\
\text { (Level 2) }\end{array}$ \\
\hline
\end{tabular}

However, the strategies that were used to remediate deficiencies in communication skills were not specified [42]. A study that surveyed how medical schools in the UK support students struggling with communication skills found that some schools had a structured remediation program that included coaching, one-onone encounters and simulated patient intervention. However, most schools used an ad hoc approach [39]. Only one study on medical students in Australia developed a remediation program based on a learning theory [47].

Based on Kirkpatrick's model of educational outcomes [32], three (18.8\%) of the studies assessed reaction, which was based on learner satisfaction and appraisal of the program [20, 33, 41, 44]. Eleven (68.8\%) assessed learning, which included changes in knowledge and skills [34, 35, 36, 37, 38, 40, 42, 43, 45-47].

\section{Discussion}

This systematic review on the remediation of deficiencies of interpersonal and communication skills of healthcare practitioners across the continuum yielded very few studies that described the diagnosis, remediation, intervention and the assessment of the outcomes of remediation. Furthermore, the studies that we identified were small scaled (range: $n=1$ to $n=225$ ) and of single-institutions. They utilized a variety of assessment methods to diagnose the specific problems the learners were struggling with including evaluations, clinical performance exams, OSCEs with SPs, direct observations, oral certifying exams and global rating scales. This is similar to the recommendations from the Kalamazoo II report that outlined specific assessment methods to evaluate communication skills [48]. Those included (i) direct observations with real patients, (ii) ratings of simulated encounters with real patients, (iii) ratings of 
Table 2 The MERSQI ${ }^{a}$ domain and item scores for the 16 selected studies that meet the review criteria

\begin{tabular}{|c|c|c|}
\hline Domain & Item & $\begin{array}{l}\text { Studies } \\
\text { N (\%) }\end{array}$ \\
\hline \multicolumn{3}{|c|}{ Study Design } \\
\hline \multicolumn{3}{|c|}{ 1.Study Design } \\
\hline & Single group cross-sectional or single group post-test only & $13(81.3)$ \\
\hline & Single group pre and post-test & $2(12.5)$ \\
\hline & Non-randomized, 2 group & $1(6.3)$ \\
\hline & Randomized controlled experiment & \\
\hline
\end{tabular}

\section{Sampling}

2. Institutions

$$
\begin{aligned}
& \text { Single institution } \\
& \text { Two institutions } \\
& \text { More than } 2 \text { institutions }
\end{aligned}
$$

3. Response Rate

$$
\begin{aligned}
& \text { Not applicable } \\
& \text { Response rate }<50 \% \text { or not reported } \\
& \text { Response rate } 50-74 \% \\
& \text { Response rate } \geq 75 \%
\end{aligned}
$$

\section{Type of Data}

4. Type of Data

$$
\begin{aligned}
& \text { Assessment by study subject } \\
& \text { Objective measurement }
\end{aligned}
$$

\section{Validity of Evaluation Instruments' Scores}

Not applicable

5. Internal Structure

$$
\text { Not reported }
$$$$
\text { Reported }
$$

6. Content

$$
\begin{aligned}
& \text { Not reported } \\
& \text { Reported }
\end{aligned}
$$

7. Relationships to other variables

$$
\begin{aligned}
& \text { Not reported } \\
& \text { Reported }
\end{aligned}
$$

\section{Data Analysis}

8. Appropriateness of analysis

Data analysis inappropriate for study design or type of data

Data analysis appropriate for study design and type of data

9. Sophistication of analysis

$$
\text { Descriptive analysis only }
$$

Beyond descriptive analysis

\section{Outcome}

10. Outcome
Satisfaction, attitudes, perceptions, opinions, general facts
Knowledge, skills

$4(26.7)$

$11(68.8)$

$16(100)$

Behaviors
Mean (SD)

$1.13(0.29)$

$\begin{array}{ll}11(68.8) & 0.5 \\ 5(31.2) & 1.5 \\ 10(62.5) & \mathrm{n} / \mathrm{a} \\ 1(6.3) & 0.5 \\ 3(18.8) & 1.5\end{array}$

$1.25(0.50)$

$2(12.5) \quad 1$

$14(87.5) \quad 3$

$9(56.3) \quad \mathrm{n} / \mathrm{a}$

$3(18.8) \quad 0$

$3(18.8) \quad 1$

$3(18.8) \quad 0$

$0.50(0.55)$

$3(18.8)$

$5(31.2) \quad 0$

$0.17(0.41)$

$1(6.3) \quad 1$

3

$2.69(0.75)$

$2.69(0.75)$

$0.50(0.55)$

$1.17(1.33)$

3

$1.0(0.0)$

$2.19(0.40)$

$1.19(0.40)$

$13(81.2) \quad 1$

$3(18.8) \quad 2$

0 
Table 2 The MERSQl ${ }^{\mathrm{a}}$ domain and item scores for the 16 selected studies that meet the review criteria (Continued)

\begin{tabular}{lllllll}
\hline Domain & Item & Studies & & Score & & Mean (SD) \\
& & Patient/health care outcome & Item & Maximum Domain & Item & Domain \\
\hline TOTAL & & 3 & 18 & $10.6(1.65)$ \\
\hline
\end{tabular}

${ }^{a}$ Medical Education Research Study Quality Instrument

video or audiotaped interactions, (iv) patient surveys and (v) examinations of knowledge, skills or attitude.

In our study, OSCE with SP was the most widely used method for assessing the learners with the majority utilizing a standardized or validated checklist. According to the literature OSCE with SPs is considered the "gold standard" tool for clinical assessment [49] as it can be designed to examine skills and ability at the "Show how" level of Miller's triangle [50]. The checklist is thought to be the most frequently used assessment tool of communication behavior as it provides clearer behavioral definitions that may improve reliability [51]. In one of the studies, the students rated practicing with SPs, receiving feedback, from SPs and faculty, in real time and observing others in small groups to be the most beneficial components of the program that helped them improve in their communication skills [41]. This was also observed in other studies that included OSCEs with opportunities for video review and feedback as part of the remediation intervention [34, 43].

Deficiencies in non-cognitive skills are the most challenging to remediate [52]. Therefore, it was not surprising that our systematic review identified a lack of standardized remediation programs for learners struggling with communication skills. However, we identified common themes for remediation strategies, which included the use of clinical practice with an SP, a clinical faculty or another peer, reflective practice, role-play, video review and structured feedback.
Having institutional policies and guidelines for remediation, a faculty development as well as a mentoring program, using learning contracts and documentation of every aspect of the remediation process are important components that support the success of the remediation plan. The challenge in the systematic review was that there were no clear outcomes specified in most of the remediation programs other than the learners progressing to the next year of their education program, passing a certifying exam or graduating.

Moreover the results from this systematic review confirm what was previously published in that there is a deficiency of outcomes-based research on strategies for remediation [23] and a lack of standardized remediation programs [53, 54]. Three steps that lead to successful remediation were identified in our study that are similar to those identified in previous studies: (i) early identification and diagnosis, (ii) developing an individualized remediation plan and (iii) reassessment and feedback $[22,26,53,55,56]$.

Our study further confirmed what was previously described in that remediation interventions lack theoretical foundation and clinical teachers struggle with using a structured process framed by appropriate theory to generate a specific educational diagnosis of learners' difficulties [55]. The majority of the studies we reviewed did not utilize theory to develop their remediation plan. Only one study used theory (socio-cognitive self-efficacy beliefs) to develop the remediation program [47] and the authors noted that

Table 3 Summary of assessment methods and remediation strategies

\begin{tabular}{|c|c|}
\hline Assessment Methods & Remediation Strategies and Methods \\
\hline $\begin{array}{l}\text { - OSCE/SP using a global rating scale } \\
\text { - OSCE/SP using a standardized checklist } \\
\text { - Structured oral interview with an OSCE } \\
\text { evaluation } \\
\text { - Direct observation of clinical encounters } \\
\text { - Direct observation of role-plays } \\
\text { - MiniCEX } \\
\text { - Monthly Evaluation } \\
\text { - } 360^{\circ} \text { evaluation } \\
\text { - Mid-point evaluation } \\
\text { - Workplace-based assessments } \\
\text { - Patient surveys } \\
\text { - Oral certifying Exams } \\
\text { - Rowland Communication Inventory }\end{array}$ & $\begin{array}{l}\text { Didactics } \\
\text { - Tutorials on communication related topics } \\
\text { - Workshops on presentation skills, doctor-patient relationships and other communication } \\
\text { topics } \\
\text { - Viewing triggers tapes } \\
\text { - Large group sessions } \\
\text { Observations } \\
\text { - OSCE Practice with SP, Clinical Faculty or other peers } \\
\text { - Direct observation of clinical encounters using global rating scale or checklist } \\
\text { - Direct observation of role-play using global rating scale or checklist } \\
\text { - Observation of faculty interacting with patients (role-modeling) followed by discussion } \\
\text { - Small group practice sessions (Clinical interviews) } \\
\text { - Performing interviews with patients } \\
\text { Reflection and Assessment } \\
\text { - One-to-one review } \\
\text { - Video Reviews followed by self-assessment and feedback } \\
\text { Other } \\
\text { - Coaching and mentoring } \\
\text { - Written tests on knowledge of communication strategies and behavioral issues } \\
\text { - Participation in CME activities }\end{array}$ \\
\hline
\end{tabular}


participants benefited from enhanced self-efficacy beliefs. Adult learning theory is thought to have a direct impact on remediation as the relevance of what is taught as well as self-direction are important since each learner has their own approach based on their life experiences [57]. Kolb's experiential learning cycle [58] has been successfully used to develop remedial courses for surgical residents struggling with the surgery-qualifying exam [57] and for residents deficient in communication skills, namely clinical interviewing skills [59]. Kolb's experiential cycle has multiple intercalations with many educational theories. Therefore designing an experiential remedial program using educational activities that mirror principles of educational theories would be beneficial [59]. Such activities would target various increasing levels of cognitive development [60], provide supportive corrective feedback [61] and reflective practice [62]. In both of the above studies, the strategies used included learning contracts, structured reflection, reviewing videos or reading material and role modeling. Although the majority of authors in the studies we reviewed do not mention the use of learning theories to develop their remediation plans they have unknowingly done so. Most of the remediation strategies used included a clinical experience (concrete experience), an observation and reflection on that experience for example reviewing the video recordings of encounters (reflective observation), conceptualizing and learning from that experience as well as learning new techniques for example through didactics and role-plays (abstract conceptualization) and finally deliberate practice to apply what was learned (active experimentation) and immediate feedback. These are the main components of Kolb's experiential cycle [58].

In our study, several remediation plans included reflection by the learners following their OSCEs, role-plays or clinical encounters with patients. Reflection before, during and after an action is foundational to self-directed learning and is necessary to promote learning. Using Schon's model [62] of the reflective practitioner provides those learners with a framework for choosing an effective action in a complex situation. It is important for the learners to be able to develop the capacity to derive lessons from a concrete clinical experience [58]. Such experiences help them refine their skills and apply their learning to subsequent encounters. By actively reflecting on what they do and do not understand, they can enhance their own learning from the concrete experience, which in turn may facilitate the potential transformative impact [63].

\section{Strengths and limitations of the review}

The major strengths of this study lie in the search process itself, which was very comprehensive and included a wide range of academic databases as well as grey literature. Additionally, we did not limit the study to one group of learners and included all healthcare practitioners across the continuum. The study however has several limitations. First, the data extraction was performed by a single author, and did not include conference abstracts, proceedings, book chapters or articles that only described an assessment tool to identify struggling learners or an intervention methodology or remediation strategy. Second based on the MERSQI score, the quality of the studies included were not high and that is a limitation of the work conducted in the area of study. Furthermore, the studies included in this systematic review were heterogeneous and hence we were not able to perform a meta-analysis. There was not enough data to indicate whether institutions that remediate trainees struggling with communication skills assess their own communication training programs to identify any deficits that could be addressed. Additionally, it was not possible to investigate the structural differences of the 16 studies identified due to the variation in the type of information provided.

\section{Implications for practice and future work}

Despite these limitations, we can make some recommendations based on our observations from the studies reviewed. Having regular evaluation and feedback methods in place may facilitate the identification of deficiencies early to avoid serious learning problems later on [64]. For a remediation strategy to be successful it is important to ensure early identification and diagnosis, the development of an individualized plan and reassessment with feedback. The most effective methods for teaching and evaluating interpersonal and communication skills involve multiple methods of assessment [3]. Therefore, we would recommend using multiple methods that would include direct observations (with patients, SPs or via video review] using a checklist or global rating scale, 360-degree evaluations, patient surveys, case discussions, role-plays or written examinations of knowledge, skills or attitude. Following the diagnosis of the problem the next steps would include discussions with the learner in order to develop an individualized remediation plan, having a learning contract, setting clear goals and objectives, a reasonable timeline, assigning a mentor, ongoing monitoring, deliberate practice, re-evaluation and feedback.

\section{Conclusion}

This study supports the need for more rigorous outcomesbased research, using control or comparison groups, for the diagnosis and remediation of healthcare practitioners struggling with interpersonal and communication skills across the continuum. It is important to consider the following practice points: (i) deficiencies in non-cognitive skills are challenging to remediate, (ii) a major challenge is whether faculty know how to identify the deficiency and what strategies to use to remediate, (iii) a variety of assessment tools need to be used to evaluate communication skills and (iv) early identification and diagnosis, creating an individualized plan and reassessment with feedback are key to successful remediation. 


\section{Appendix}

\section{Literature search}

Table 4 Academic databases

\begin{tabular}{|c|c|c|c|}
\hline $\begin{array}{l}\text { Source and } \\
\text { search date }\end{array}$ & Search String & Result & Notes \\
\hline $\begin{array}{l}\text { PubMed } \\
\text { Search } \\
\text { Date: } 2018- \\
06-08\end{array}$ & $\begin{array}{l}\text { (("psychiatrists"[Title/Abstract] OR "psychiatrist"[Title/Abstract] OR "premedical } \\
\text { education"[Title/Abstract] OR "dental education"[Title/Abstract] OR "dental } \\
\text { educations" [Title/Abstract] OR "pharmaceutical education"[Title/Abstract] OR } \\
\text { "pharmaceutical education" Title/Abstract] OR "pharmaceutical school" Title/ }\end{array}$ & 232 & $\begin{array}{l}\text { All terms searched in field "Title/Abstract" and } \\
\text { in "MeSH" when available. } \\
\text { Filter for publication year applied }\end{array}$ \\
\hline
\end{tabular}

Coverage: Abstract] OR "pharmaceutical schools" [Title/Abstract] OR "pharmaceutical student" [Title/Abstract] OR "pharmaceutical students" [Title/Abstract] OR "pharmacy education" [Title/Abstract] OR "pharmacy educations" [Title/Abstract] OR "nursing school"[Title/Abstract] OR "nursing schools"[Title/Abstract] OR "dental school"[Title/Abstract] OR "dental schools"[Title/Abstract] OR ("pharmacy school"[Title/Abstract] OR "pharmacy schools"[Title/Abstract] OR physiotherapist [Title/Abstract] OR physiotherapists [Title/Abstract] OR "physiotherapy education"[Title/Abstract] OR "physiotherapy students"[Title/ Abstract] OR "physiotherapy student"[Title/Abstract] OR "physiotherapy school"[Title/Abstract] OR "physiotherapy schools"[Title/Abstract] OR "physical therapy faculty"[Title/Abstract] OR "physiotherapy faculty"[Title/Abstract] OR "physical therapy student"[Title/Abstract] OR "physical therapy students"[Title/ Abstract] OR "physical therapy education"[Title/Abstract] OR "physical therapy educations"[Title/Abstract] OR "physical therapy school"[Title/Abstract] OR "physical therapy schools"[Title/Abstract] OR "Schools, Dental"[Mesh] OR "Schools, Nursing"[Mesh] OR "Schools, Pharmacy"[Mesh] OR "Education, Pharmacy"[Mesh] .OR "Education, Premedical"[Mesh]/ OR "Education, Dental"[Mesh] OR "Education, Nursing"[Mesh] OR "medical curricula"[Title/ Abstract] OR "medical curriculum"[Title/Abstract] OR "medical faculty"[Title/ Abstract] OR "medical learners"[Title/Abstract] OR interns [Title/Abstract] OR intern [Title/Abstract] OR internship [Title/Abstract] OR internships [Title/ Abstract] OR "Education, Medical"[Mesh] OR "Internship and Residency"[Mesh] OR "Clinical Clerkship"[Mesh] OR "Students, Medical"[Mesh] OR "medical postgraduates"[Title/Abstract] OR "medical postgraduate"[Title/Abstract] OR "medical graduate"[Title/Abstract] OR "medical graduates"[Title/Abstract] OR "medical undergraduate"[Title/Abstract] OR "medical undergraduates"[Title/ Abstract] OR "medical student"[Title/Abstract] OR "medical students"[Title/ Abstract] OR "medical education"[Title/Abstract] OR "medical educations"[Title/Abstract] OR clerkship*[Title/Abstract] OR resident [Title/ Abstract] OR residents [Title/Abstract] OR residency [Title/Abstract] OR "teaching round"[Title/Abstract] OR "teaching rounds"[Title/Abstract] OR "Health Personnel"[Mesh] OR "medical school"[Title/Abstract] OR "medical schools"[Title/Abstract] OR "medical college"[Title/Abstract] OR "medical universities"[Title/Abstract] OR "medical university"[Title/Abstract] OR "Schools, Medical"[Mesh] OR "physical therapists" [Title/Abstract] OR "physical therapist" [Title/Abstract] OR physician*[Title/Abstract] OR pharmacist* [Title/Abstract] OR nurse*[Title/Abstract] OR "medical staff"[Title/Abstract] OR "health educators" [Title/Abstract] OR "nursing faculty"[Title/Abstract] OR "dental faculty"[Title/Abstract] OR dentist*[Title/Abstract] OR "pharmacy faculty"[Title/ Abstract] OR "health personnel" [Title/Abstract] OR "allied health personnel"[Title/Abstract] OR "Faculty, Nursing"[Mesh] OR "Faculty, Medical"[Mesh] OR "Faculty, Dental"[Mesh] OR "Students, Nursing"[Mesh] OR "Students, Pharmacy"[Mesh] OR "Students, Dental"[Mesh] OR "Students, Premedical"[Mesh] OR "premedical student"[Title/Abstract] OR "premedical students"[Title/Abstract] OR "pharmacy students" [Title/Abstract] OR "pharmacy student"[Title/Abstract] OR "dental student"[Title/Abstract] OR "dental students"[Title/Abstract] OR "nursing students"[Title/Abstract] OR "Nursing education"[Title/Abstract] OR "Nursing educations"[Title/Abstract] OR "nursing student"[Title/Abstract] OR "pharmacy graduates"[Title/Abstract] OR "pharmacy undergraduates"[Title/Abstract] OR "pharmacy graduate"[Title/ Abstract] OR "pharmacy undergraduate" [Title/Abstract] OR "pharmacy postgraduates"[Title/Abstract] OR "pharmacy postgraduate"[Title/Abstract] OR "nursing graduates"[Title/Abstract] OR "nursing graduate"[Title/Abstract] OR "nursing undergraduates"[Title/Abstract] OR "nursing undergraduates"[Title/ Abstract] OR "dental graduates"[Title/Abstract] OR "dental graduate"[Title/ Abstract] OR "dental undergraduates"[Title/Abstract] OR "dental undergraduate"[Title/Abstract] OR "dental postgraduates"[Title/Abstract] OR "dental postgraduate"[Title/Abstract]) AND ("Remedial Teaching"[Mesh] OR Remediation*[Title/Abstract] OR remedial* [Title/Abstract]) AND ("Teach-Back Communication"[Mesh] OR "Health Communication"[Mesh] OR 
Table 4 Academic databases (Continued)

\begin{tabular}{ll}
\hline $\begin{array}{l}\text { Source and } \\
\text { search date }\end{array}$ & Search String \\
\hline & "Interdisciplinary Communication"[Mesh] OR "Persuasive \\
& Communication"[Mesh] OR "Nonverbal Communication"[Mesh] OR \\
"Communication Barriers"[Mesh] OR "communication"[Mesh] OR \\
communicat*[Title/Abstract] OR "interpersonal relation"[Title/Abstract] OR \\
"interpersonal relationships" [Title/Abstract] OR "interpersonal relationship" \\
[Title/Abstract] OR "interpersonal relations"[Title/Abstract] OR "interpersonal \\
Interaction" [Title/Abstract] OR "interpersonal Interactions" [Title/Abstract] OR \\
"Interpersonal Relations"[Mesh]))
\end{tabular}

Scopus

Search

Date: 2018

06-08

Coverage:

1960-

(TITLE-ABS-KEY ("psychiatrists" OR "psychiatrist" OR "premedical education" OR

"dental education" OR "dental educations" OR "pharmaceutical education" OR

"pharmaceutical education" OR "pharmaceutical school" OR "pharmaceutical schools" OR "pharmaceutical student" OR "pharmaceutical students" OR

"pharmacy educations" OR "nursing school" OR "nursing schools" OR "dental school" OR "dental schools" OR "pharmacy school" OR "pharmacy schools" OR physiotherapist OR physiotherapists OR "physiotherapy education" OR "physiotherapy students" OR "physiotherapy student" OR "physiotherapy school" OR "physiotherapy schools" OR "physical therapy faculty" OR "physiotherapy faculty" OR "physical therapy student" OR "physical therapy students" OR "physical therapy education" OR "physical therapy educations" OR "physical therapy school" OR "physical therapy schools" OR "medical curricula" OR "medical curriculum" OR "medical faculty" OR "medical learners" OR interns OR intern OR internship OR internships OR "medical postgraduates" OR "medical postgraduate" OR "medical graduate" OR "medical graduates" OR "medical undergraduate" OR "medical undergraduates" OR "medical student" OR "medical students" OR "medical education" OR "medical educations" OR clerkship* OR residents OR residency OR "teaching round" OR "teaching rounds" OR "health Personnel" OR "medical school" OR "medical schools" OR "Medical college" OR "medical universities" OR "medical university" OR "physical therapists"

OR "physical therapist" OR physician* OR pharmacist*

OR nurse* OR "medical staff" OR "health educators" OR "nursing faculty" OR "dental faculty" OR dentist* OR "pharmacy faculty" OR "health personnel" OR "allied health personnel"

OR "premedical student" OR "premedical students" OR "pharmacy students" OR "pharmacy student" OR "dental student" OR "dental students" OR "nursing students" OR "nursing education" OR "nursing educations" OR "nursing student" OR "pharmacy graduates"

OR "pharmacy undergraduates" OR "pharmacy graduate"

OR "pharmacy undergraduate" OR "pharmacy postgraduates" OR "pharmacy postgraduate" OR "nursing graduates" OR "nursing graduate" OR "nursing undergraduates" OR "nursing undergraduates" OR "dental graduates" OR "dental graduate" OR "dental undergraduates" OR "dental undergraduate" OR "dental postgraduates" OR "dental postgraduate") AND (TITLE-ABS-KEY (communicat* OR "interpersonal relation" OR "interpersonal relationships" OR "interpersonal relationship" OR "interpersonal relations" OR "interpersonal interaction" OR "interpersonal interactions") AND (TITLE-ABS-KEY (remediation* OR remedial*))

Web of

Science

Search

Date: 2018

06-08

Coverage:

1864-

Result Notes

233 for "Title", Abstract" and "Keywords", here marked with "TI-ABS-KEY."

No thesaurus available.

No filters or limitations applied
191 - All terms searched in the field "Topic" (which includes the fields: "Abstract", "Title", Author Keywords and "Keyword Plus").

- Title and abstract search only is not available. No thesaurus available. No filters or limitations applied 
Table 4 Academic databases (Continued)

\begin{tabular}{ll}
\hline $\begin{array}{l}\text { Source and } \\
\text { search date }\end{array}$ & Search String \\
\hline & OR "teaching round" OR "teaching rounds" OR "Health Personnel" OR \\
"medical school" OR "medical schools" OR "Medical college" OR "medical \\
universities" OR "medical university" OR "Physical therapists" OR "Physical \\
therapist" OR physician* OR pharmacist* OR nurse* OR "medical staff" OR \\
"health educators" OR "nursing faculty" OR "dental faculty" OR dentist* OR \\
"pharmacy faculty" OR "health personnel" OR "allied health personnel" OR \\
"premedical student" OR "premedical students" OR "pharmacy students" OR \\
"pharmacy student" OR "dental student" OR "dental students" OR "nursing \\
students" OR "Nursing education "OR "Nursing educations "OR "nursing \\
student" OR "pharmacy graduates" OR "pharmacy undergraduates" OR \\
"pharmacy graduate" OR "pharmacy undergraduate" OR "pharmacy \\
postgraduates" OR "pharmacy postgraduate" OR "nursing graduates" OR \\
"nursing graduate" OR "nursing undergraduates" OR "nursing undergraduates" \\
OR "dental graduates" OR "dental graduate" OR "dental undergraduates" OR \\
"dental undergraduate" OR "dental postgraduates" OR "dental postgraduate") \\
AND (Remediation* OR remedial*) AND (communicat* OR "interpersonal \\
relation" OR "Interpersonal relationships" OR "Interpersonal relationship" OR \\
"interpersonal relations" OR "Interpersonal Interaction" OR "Interpersonal \\
Interactions"))
\end{tabular}

Psych- Info TI/AB (("psychiatrists" OR "psychiatrist" OR "premedical education" OR "dental (EBSCO)

Search

Date: 2018

06-08

Coverage:

1964-
Result Notes

All terms searched in the fields "Title and "Abstract" (here marked as TI/AB) and in the thesaurus (DE), when available. No filters or limitations applied 
Table 4 Academic databases (Continued)

\begin{tabular}{|c|c|c|c|}
\hline $\begin{array}{l}\text { Source and } \\
\text { search date }\end{array}$ & Search String & Result & Notes \\
\hline $\begin{array}{l}\text { CINAHL } \\
\text { (EBSCO) } \\
\text { Search } \\
\text { Date: } \\
\text { 2018-06-08 }\end{array}$ & $\begin{array}{l}\text { Tl/AB (("psychiatrists" OR "psychiatrist" OR "premedical education" OR "dental } \\
\text { education" OR "dental educations" OR "pharmacy education" OR "pharmacy } \\
\text { educations" OR "nursing school" OR "nursing schools" OR "dental school" OR } \\
\text { "dental schools" OR "pharmacy school" OR "pharmacy schools" OR } \\
\text { "physiotherapist" OR "physiotherapists" OR "physiotherapy education" OR }\end{array}$ & 51 & $\begin{array}{l}\text { All terms searched in the fields "Title" (".ti"), } \\
\text { "Abstract" (".ab") and in the "Thesaurus" ("/") } \\
\text { when available. } \\
\text { No filters or limitations applied }\end{array}$ \\
\hline
\end{tabular}

Coverage: "physiotherapy students" OR "physiotherapy student" OR "physiotherapy

1981-

school" OR "physiotherapy schools" OR "physical therapy faculty" OR

"physiotherapy faculty" OR "physical therapy student" OR "physical therapy students" OR "physical therapy education" OR "physical therapy educations" OR "physical therapy school" OR "physical therapy schools" OR "medical curricula" OR "medical curriculum" OR "medical faculty" OR "medical learners" OR interns OR intern OR internship OR internships OR "medical postgraduates" OR "medical postgraduate" OR "medical graduate" OR "medical graduates" OR "medical undergraduate" OR "medical undergraduates" OR "medical student" OR "medical students" OR "medical education" OR "medical educations" OR clerkship* OR residents OR residency OR "teaching round" OR "teaching rounds" OR "health Personnel" OR "medical school" OR "medical schools" OR "medical college" OR "medical universities" OR "medical university" OR "physical therapists" OR "physical therapist" OR "physiotherapist" OR "physiotherapists" OR physician* OR pharmacist* OR nurse* OR "medical staff" OR "health educators" OR "nursing faculty" OR "dental faculty" OR dentist* OR "pharmacy faculty" OR "health personnel" OR "allied health personnel" OR "premedical student" OR "premedical students" OR "pharmacy students" OR "pharmacy student" OR "dental student" OR "dental students" OR "nursing students" OR "nursing education "OR "nursing educations "OR "nursing student" OR "pharmacy graduates" OR "pharmacy undergraduates" OR "pharmacy graduate" OR "pharmacy undergraduate" OR "pharmacy postgraduates" OR "pharmacy postgraduate" OR "nursing graduates" OR "nursing graduate" OR "nursing undergraduates" OR "nursing undergraduates" OR "dental graduates" OR "dental graduate" OR "dental undergraduates" OR "dental undergraduate" OR "dental postgraduates" OR "dental postgraduate" OR "pharmaceutical education" OR "pharmaceutical education" OR "pharmaceutical school" OR "pharmaceutical schools" OR "pharmaceutical student" OR "pharmaceutical students") OR MH ("Health Personnel" OR "Allied Health Personnel" OR "Dentists" OR "Nurses" OR "Physicians"

"Pharmacists" OR "Faculty, Dental" OR "Education, Medical" OR "Education, Medical, Continuing" OR "Schools, Medical" OR "Internship and Residency" OR "Interns and Residents" OR "Students, Medical"

OR "Students, Dental" OR "Students, Pharmacy" OR "Faculty, Medical" OR "Faculty, Nursing" OR "Medical Staff" OR "Pharmacists" OR "Physical Therapists" OR "Schools, Dental" OR "Education, Dental" OR "Psychiatrists" OR "Education, Nursing" OR "Education, Nursing, Diploma Programs" OR "Education, Nursing, Practical" OR "Education, Nursing, Masters" OR "Education, Nursing, TheoryBased" OR "Education, Nursing, Research-Based" OR "Education, Nursing, Graduate" OR "Education, Nursing, Continuing" OR "Education, Nursing, Baccalaureate" OR "Students, Nursing, Male" OR "Students, Nursing, Graduate" OR "Students, Nursing, Masters" OR "Students, Nursing, Diploma Programs" OR "Students, Nursing, Baccalaureate" OR "Students, Nursing, Practical" OR "Schools, Nursing" OR "Education, Nursing, Diploma Programs" OR "School Health Nursing" OR "Education, Pharmacy" OR "Education, Premedical") AND TI/AB (communicat* OR "interpersonal relation" OR "interpersonal relationships" OR "interpersonal relationship" OR "interpersonal relations" OR "interpersonal Interaction" OR "interpersonal interactions") OR MH ("Interpersonal Relations" OR "Communication" OR "Nonverbal Communication" OR "Communication Skills Training" OR "Communication Skills" OR "Communication Barriers" OR "Persuasive Communication") AND TI/AB (remediation* OR remedial*) OR MH ("Remedial Teaching"))

EMBASE (OVID)

Search

Date: 2018-

06-10

Coverage:

1974-
TI/AB (("psychiatrists" OR "psychiatrist" OR "premedical education" OR "dental education" OR "dental educations" OR "pharmacy education" OR "pharmacy educations" OR "nursing school" OR "nursing schools" OR "dental school" OR "dental schools" OR "pharmacy school" OR "pharmacy schools" OR "physiotherapist" OR "physiotherapists" OR "physiotherapy education" OR "physiotherapy students" OR "physiotherapy student" OR "physiotherapy school" OR "physiotherapy schools" OR "physical therapy faculty" OR "physiotherapy faculty" OR "physical therapy student" OR "physical therapy students" OR "physical therapy education" OR "physical therapy educations"
All terms searched in the fields "Title" (".ti"), "Abstract" (".ab") and in the "Thesaurus" ("/") when available.

No filters or limitations applied

"Remediation" not included in the thesaurus. The thesaurus term: "communication disorder" included in the search here as it refers to "communication barriers" etc. 
Table 4 Academic databases (Continued)

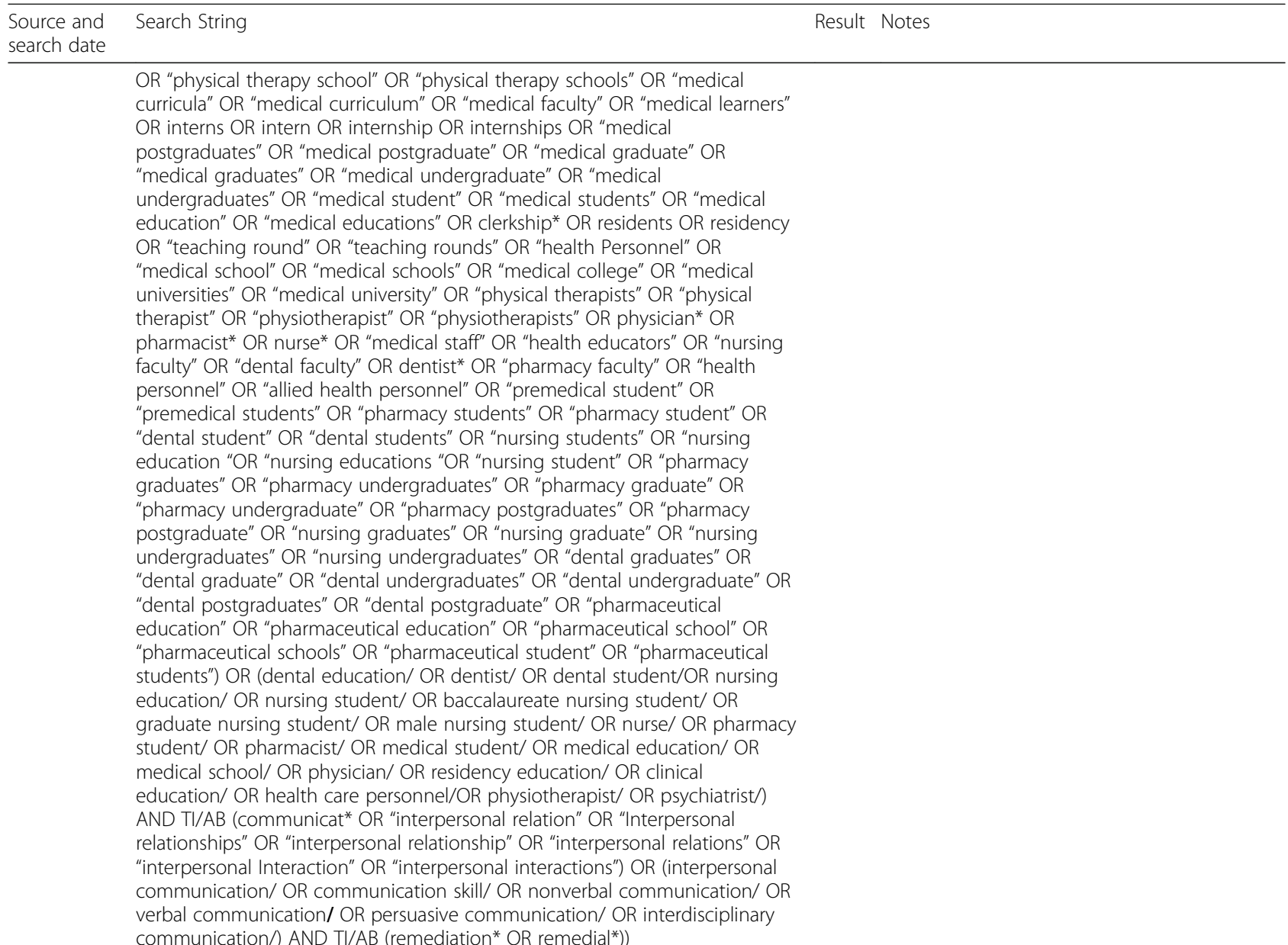

ERIC (OVID) TI/AB ("psychiatrists" OR "psychiatrist" OR "premedical education" OR "dental

\section{Search}

Date:

2018-06-07

Coverage:

1965education" OR "dental educations" OR "pharmacy education" OR "pharmacy educations" OR "nursing school" OR "nursing schools" OR "dental school" OR "dental schools" OR "pharmacy school" OR "pharmacy schools" OR "physiotherapist" OR "physiotherapists" OR "physiotherapy education" OR "physiotherapy students" OR "physiotherapy student" OR "physiotherapy school" OR "physiotherapy schools" OR "physical therapy faculty" OR "physiotherapy faculty" OR "physical therapy student" OR "physical therapy students" OR "physical therapy education" OR "physical therapy educations" OR "physical therapy school" OR "physical therapy schools" OR "medical curricula" OR "medical curriculum" OR "medical faculty" OR "medical learners" OR interns OR intern OR internship OR internships OR "medical postgraduates" OR "medical postgraduate" OR "medical graduate" OR "medical graduates" OR "medical undergraduate" OR "medical undergraduates" OR "medical student" OR "medical students" OR "medical education" OR "medical educations" OR clerkship* OR residents OR residency OR "teaching round" OR "teaching rounds" OR "health Personnel" OR "medical school" OR "medical schools" OR "medical college" OR "medical universities" OR "medical university" OR "physical therapists" OR "physical therapist" OR "physiotherapist" OR "physiotherapists" OR physician* OR pharmacist* OR nurse* OR "medical staff" OR "health educators" OR "nursing faculty" OR "dental faculty" OR dentist* OR "pharmacy faculty" OR "health personnel" OR "allied health personnel" OR "premedical student" OR "premedical students" OR "pharmacy students" OR "pharmacy student" OR "dental student" OR "dental students" OR "nursing students" OR "nursing education "OR "nursing educations "OR "nursing student" OR "pharmacy graduates" OR "pharmacy undergraduates" OR "pharmacy graduate" OR "pharmacy undergraduate" OR "pharmacy postgraduates" OR "pharmacy postgraduate" OR "nursing graduates" OR "nursing graduate" OR "nursing undergraduates" OR "nursing undergraduates" OR "dental graduates" OR

All terms searched in the fields "Title" (".ti"), "Abstract" (".ab") and in the "Thesaurus" ("/") when available.

No filters or limitations applied 
Table 4 Academic databases (Continued)

Source and Search String

"dental graduate" OR "dental undergraduates" OR "dental undergraduate" OR

"dental postgraduates" OR "dental postgraduate" OR "pharmaceutical education" OR "pharmaceutical education" OR "pharmaceutical school" OR "pharmaceutical schools" OR "pharmaceutical student" OR "pharmaceutical students") OR (dental schools/ OR dentistry/ OR nursing students/ OR allied health occupations education/ OR nurses/ OR nursing education/ OR medical schools/

OR graduate medical education/ OR medical education/ OR medical students/ OR medical schools/ OR graduate medical education/ OR medical education/ OR medical school faculty/ OR medical students/ OR pharmacy/ OR pharmaceutical education/ OR premedical students/ OR undergraduate students/ OR medical education/ OR medical students/ OR nursing education/ OR clinical experience/ OR health personnel/ OR allied health personnel/ OR nurses/ OR physicians/ OR psychologists/ OR nursing education/ OR pharmaceutical education/) AND TI/AB (communicat* OR "interpersonal relation" OR "Interpersonal relationships" OR "interpersonal relationship" OR "interpersonal relations" OR "interpersonal Interaction" OR "interpersonal interactions") OR (verbal communication/ OR nonverbal communication/ OR interpersonal relationship/) AND TI/AB (remediation* OR remedial*) OR (remedial instruction/)) 
Table 5 Updated search in PubMed and scopus, 2019-05-14

\begin{tabular}{|c|c|c|c|}
\hline $\begin{array}{l}\text { Source } \\
\text { and search } \\
\text { date }\end{array}$ & Search String & Result & Notes \\
\hline $\begin{array}{l}\text { PubMed } \\
\text { Search } \\
\text { Date: } \\
\text { 2019-05-14 }\end{array}$ & $\begin{array}{l}\text { ((“"psychiatrists"[Title/Abstract] OR "psychiatrist"[Title/Abstract] OR } \\
\text { "premedical education"[Title/Abstract] OR "dental education"[Title/ } \\
\text { Abstract] OR "dental educations" [Title/Abstract] OR "pharmaceutical } \\
\text { education"[Title/Abstract] OR "pharmaceutical education" [Title/ } \\
\text { Abstract] OR "pharmaceutical school" [Title/Abstract] OR }\end{array}$ & 12 & $\begin{array}{l}\text { All terms searched in field "Title/Abstract" and in "MeSH" when } \\
\text { available. } \\
\text { Filter for publication year, 2018-06-08- 2019-05-14 applied } \\
\text { No duplicates } \\
\text { detected within the result }\end{array}$ \\
\hline
\end{tabular}

"pharmaceutical schools" [Title/Abstract] OR "pharmaceutical student" [Title/Abstract] OR "pharmaceutical students" [Title/Abstract] OR "pharmacy education"[Title/Abstract] OR "pharmacy educations"[Title/ Abstract] OR "nursing school"[Title/Abstract] OR "nursing schools" [Title/Abstract] OR "dental school"[Title/Abstract] OR "dental schools"[Title/Abstract] OR ("pharmacy school"[Title/Abstract] OR "pharmacy schools"[Title/Abstract] OR physiotherapist [Title/Abstract] OR physiotherapists [Title/Abstract] OR "physiotherapy education"[Title/Abstract] OR "physiotherapy students"[Title/Abstract] OR "physiotherapy student" [Title/Abstract] OR "physiotherapy school"Title/Abstract] OR "physiotherapy schools"[Title/Abstract] OR "physical therapy faculty"[Title/Abstract] OR "physiotherapy faculty"[Title/Abstract] OR "physical therapy student"[Title/Abstract] OR "physical therapy students"[Title/Abstract] OR "physical therapy education"[Title/Abstract] OR "physical therapy educations"[Title/ Abstract] OR "physical therapy school"[Title/Abstract] OR "physical therapy schools"[Title/Abstract] OR "Schools, Dental"[Mesh] OR "Schools, Nursing"[Mesh] OR "Schools, Pharmacy"[Mesh] OR "Education, Pharmacy"[Mesh] OR "Education, Premedical"[Mesh] OR "Education, Dental"[Mesh] OR "Education, Nursing"[Mesh] OR "medical curricula"[Title/Abstract] OR "medical curriculum"[Title/Abstract] OR "medical faculty"[Title/Abstract] OR "medical learners"[Title/Abstract] OR interns [Title/Abstract] OR intern [Title/Abstract] OR internship [Title/Abstract] OR internships [Title/Abstract] OR "Education, Medical"[Mesh] OR "Internship and Residency"[Mesh] OR "Clinical Clerkship"[Mesh] OR "Students, Medical"[Mesh] OR "medical postgraduates"[Title/Abstract] OR "medical postgraduate"[Title/ Abstract] OR "medical graduate"[Title/Abstract] OR "medical graduates"[Title/Abstract] OR "medical undergraduate"[Title/Abstract] OR "medical undergraduates"[Title/Abstract] OR "medical student"[Title/Abstract] OR "medical students"[Title/Abstract] OR "medical education"Title/Abstract] OR "medical educations"[Title/ Abstract] OR clerkship*[Title/Abstract] OR resident [Title/Abstract] OR residents [Title/Abstract] OR residency [Title/Abstract] OR "teaching round" $[$ itle/Abstract] OR "teaching rounds" [Title/Abstract] OR "Health Personnel"[Mesh] OR "medical school"[Title/Abstract] OR "medical schools"[Title/Abstract] OR "medical college"[Title/Abstract] OR "medical universities"[Title/Abstract] OR "medical university"[Title/ Abstract] OR "Schools, Medical"[Mesh] OR "physical therapists" [Title/ Abstract] OR "physical therapist" [Title/Abstract] OR physician*[Title/ Abstract] OR pharmacist*[Title/Abstract] OR nurse*[Title/Abstract] OR "medical staff"[Title/Abstract] OR "health educators"[Title/Abstract] OR "nursing faculty"[Title/Abstract] OR "dental faculty"[Title/Abstract] OR dentist*[itle/Abstract] OR "pharmacy faculty"[Title/Abstract] OR "health personnel"[Title/Abstract] OR "allied health personnel" [Title/ Abstract] OR "Faculty, Nursing"[Mesh] OR "Faculty, Medical"[Mesh] OR "Faculty, Dental"[Mesh] OR "Students, Nursing"[Mesh] OR "Students, Pharmacy"[Mesh] OR "Students, Dental"[Mesh] OR "Students, Premedical"[Mesh] OR "premedical student"[Title/Abstract] OR "premedical students"[Title/Abstract] OR "pharmacy students"[Title/ Abstract] OR "pharmacy student" [Title/Abstract] OR "dental student"[Title/Abstract] OR "dental students"[Title/Abstract] OR "nursing students"[Title/Abstract] OR "Nursing education"[Title/ Abstract] OR "Nursing educations"[Title/Abstract] OR "nursing student"[Title/Abstract] OR "pharmacy graduates"[Title/Abstract] OR "pharmacy undergraduates" [Title/Abstract] OR "pharmacy graduate"[Title/Abstract] OR "pharmacy undergraduate"[Title/Abstract] OR "pharmacy postgraduates"[Title/Abstract] OR "pharmacy postgraduate"[Title/Abstract] OR "nursing graduates"[Title/Abstract] OR "nursing graduate"[Title/Abstract] OR "nursing undergraduates"[Title/Abstract] OR "nursing undergraduates" [Title/ Abstract] OR "dental graduates"[Title/Abstract] OR "dental graduate"[Title/Abstract] OR "dental undergraduates"[Title/Abstract] OR "dental undergraduate"[Title/Abstract] OR "dental postgraduates"[Title/Abstract] OR "dental postgraduate"[Title/ 
Table 5 Updated search in PubMed and scopus, 2019-05-14 (Continued)

\begin{tabular}{ll}
\hline $\begin{array}{l}\text { Source } \\
\text { and search } \\
\text { date }\end{array}$ & Search String \\
\hline & \\
& Abstract]) AND ("Remedial Teaching"[Mesh] OR Remediation*[Title/ \\
& Abstract] OR remedial* [Title/Abstract]) AND ("Teach-Back \\
& Communication"[Mesh] OR "Health Communication"[Mesh] OR \\
& "Interdisciplinary Communication"[Mesh] OR "Persuasive \\
& Communication"[Mesh] OR "Nonverbal Communication"[Mesh] OR \\
& "Communication Barriers"[Mesh] OR "communication"[Mesh] OR \\
& communicat*[Title/Abstract] OR "interpersonal relation"[Title/Abstract] \\
& OR "interpersonal relationships" [Title/Abstract] OR "interpersonal \\
& relationship" [Title/Abstract] OR "interpersonal relations"[Title/Abstract] \\
& OR "interpersonal Interaction" [Title/Abstract] OR "interpersonal \\
Interactions" [Title/Abstract] OR "Interpersonal Relations"[Mesh])))
\end{tabular}

Scopus

Search

Date:

2019-05-14
(TITLE-ABS-KEY ("psychiatrists" OR "psychiatrist" OR "premedical education" OR "dental education" OR "dental educations" OR "pharmaceutical education" OR "pharmaceutical education" OR "pharmaceutical school" OR "pharmaceutical schools" OR "pharmaceutical student" OR "pharmaceutical students" OR "pharmacy educations" OR "nursing school" OR "nursing schools" OR "dental school" OR "dental schools" OR "pharmacy school" OR "pharmacy schools" OR physiotherapist OR physiotherapists OR "physiotherapy education" OR "physiotherapy students" OR "physiotherapy student" OR "physiotherapy school" OR "physiotherapy schools" OR "physical therapy faculty" OR "physiotherapy faculty" OR "physical therapy student" OR "physical therapy students" OR "physical therapy education" OR "physical therapy educations" OR "physical therapy school" OR "physical therapy schools" OR "medical curricula" OR "medical curriculum" OR "medical faculty" OR "medical learners" OR interns OR intern OR internship OR

internships OR "medical postgraduates" OR "medical postgraduate" OR "medical graduate" OR "medical graduates" OR "medical undergraduate" OR "medical undergraduates" OR "medical student" OR "medical students" OR "medical education" OR "medical educations" OR clerkship* OR residents OR residency OR "teaching round" OR "teaching rounds" OR "health Personnel" OR "medical school" OR "medical schools" OR "Medical college" OR "medical universities" OR "medical university" OR "physical therapists" OR "physical therapist" OR physician* OR pharmacist* OR nurse* OR "medical staff" OR "health educators" OR "nursing faculty" OR "dental faculty" OR dentist* OR "pharmacy faculty" OR "health personnel" OR "allied health personnel"

OR "premedical student" OR "premedical students" OR "pharmacy students" OR "pharmacy student" OR "dental student" OR "dental students" OR "nursing students" OR "nursing education" OR "nursing educations" OR "nursing student" OR "pharmacy graduates" OR "pharmacy undergraduates" OR "pharmacy graduate" OR "pharmacy undergraduate" OR "pharmacy postgraduates" OR "pharmacy postgraduate" OR "nursing graduates" OR "nursing graduate" OR "nursing undergraduates" OR "nursing undergraduates" OR "dental graduates" OR "dental graduate" OR "dental undergraduates" OR "dental undergraduate" OR "dental postgraduates" OR "dental postgraduate") AND (TITLE-ABS-KEY (communicat* OR "interpersonal relation" OR "interpersonal relationships" OR "interpersonal relationship" OR "interpersonal relations" OR "interpersonal interaction" OR "interpersonal interactions") AND (TITLE-ABS-KEY (remediation* OR remedial*))

Total numbers of references

Duplicates removed

Total numbers of references after de-duplication
All selected search terms searched in the fields for "Title", Abstract" and "Keywords", here marked with "TI-ABS-KEY."

No thesaurus available

Filter for publication year, 2018- the search date were applied. The references form 2018 were screened by hand to only include studies published after 2018-06-08- in the result.

No duplicates

detected within the result 
Table 6 Grey sources

Source and search date

ProQuest Dissertation and Theses

Search date: $2019-05-18$

\author{
Search string
}

((("psychiatrists" OR "psychiatrist" OR "premedical education" OR "dental 34 education" OR "dental educations" OR "pharmaceutical" OR "pharmacy educations" OR "nursing school" OR "nursing schools" OR "dental school" OR "dental schools" OR "pharmacy school" OR "pharmacy schools" OR physiotherapist OR physiotherapists OR "physiotherapy education" OR "physiotherapy students" OR "physiotherapy student" OR "physiotherapy school" OR "physiotherapy schools" OR "physical therapy faculty" OR "physiotherapy faculty" OR "physical therapy student" OR "physical therapy students" OR "physical therapy education" OR "physical therapy educations" OR "physical therapy school" OR "physical therapy schools" OR "medical curricula" OR "medical curriculum" OR "medical faculty" OR "medical learners" OR interns OR intern OR internship OR internships OR "medical postgraduates" OR "medical postgraduate" OR "medical graduate" OR "medical graduates" OR "medical undergraduate" OR "medical undergraduates" OR "medical student" OR "medical students" OR "medical education" OR "medical educations" OR clerkship* OR residents OR residency OR "teaching round" OR "teaching rounds" OR "health personnel" OR "medical school" OR "medical schools" OR "medical college" OR "medical universities" OR "medical university" OR "physical therapists" OR "physical therapist" OR physician* OR pharmacist* OR nurse* OR "medical staff" OR "health educators" OR "nursing faculty" OR "dental faculty" OR dentist* OR "pharmacy faculty" OR "health personnel" OR "allied health personnel" OR "premedical student" OR "premedical students" OR "pharmacy students" OR "pharmacy student" OR "dental student" OR "dental students" OR "nursing students" OR "nursing education" OR "nursing educations" OR "nursing student" OR "pharmacy graduates" OR "pharmacy undergraduates" OR "pharmacy graduate" OR "pharmacy undergraduate" OR "pharmacy postgraduates" OR "pharmacy postgraduate" OR "nursing graduates" OR "nursing graduate" OR "nursing undergraduates" OR "nursing undergraduates" OR "dental graduates" OR "dental graduate" OR "dental undergraduates" OR "dental undergraduate" OR "dental postgraduates" OR "dental postgraduate") AND (remediation* OR remedial*) AND (communicat* OR "interpersonal relation" OR "interpersonal relationships" OR "interpersonal relationship" OR "interpersonal relations" OR "interpersonal Interaction" OR "interpersonal interactions")))

Ethos

Search date: $2019-05-18$

remediation AND communication AND student

remediation AND communication AND medical

remediation AND communication AND physicians

remediation AND interpersonal AND medical

remediation AND interpersonal

Open Grey

(Grey literature in Europe)

Search date: 2019-05-18

BASE

(Bielefeld Academic

Search Engine)

Search date: 2019-05-18

The New York Academy of Grey Literature

Reports

Search date: $2019-05-18$

British Library Main remediation AND communication

remediation AND communication AND students

remediation AND communication AND medical

remediation AND interpersonal AND medical

remediation AND interpersonal

remediation AND communication AND physicians

tit:communicat* tit:remedia*

subj:remedia* subj:communica*

subj:remedia* subj:communica* subj:medical*

subj:interpersonal* subj:remedia* subj:medical*

subj:interpersonal* subj:remedia* subj:student*

remediation AND communication

remediation AND interpersonal

remediation AND students

remedia* AND communica*

Result Notes

Search in "All fields Except Full text".

No filters or limitations applied

* The search function in this source is very limited. A broad search was conducted. No filters or limitations applied.

* The search function in this source is very limited. A broad search was conducted.

Searched in the field for "Title"

Searched in the field for "Subject"

Searched with publication limitation: 
Table 6 Grey sources (Continued)

\begin{tabular}{|c|c|c|c|}
\hline Source and search date & Search string & Result & Notes \\
\hline \multirow{5}{*}{$\begin{array}{l}\text { Catalogue } \\
\text { Search date: } 2019-05-18\end{array}$} & remedia* AND communica* $^{*}$ AND medical ${ }^{*}$ & 0 & "Theses" \\
\hline & remedia* AND communica* AND medical* & 81 & \multirow[t]{4}{*}{ Searched in "Whole Catalogue" } \\
\hline & remedia* AND interpersonal* AND medical* & 10 & \\
\hline & remedia* AND interpersonal* & 104 & \\
\hline & remedia* AND interpersonal* AND student* & 11 & \\
\hline \multirow{2}{*}{$\begin{array}{l}\text { WorldCat library } \\
\text { catalogue } \\
\text { Search date: } 2019-05-18\end{array}$} & ti:remedia* ti:communicat* $^{*}$ ti:medical ${ }^{*}$ & 21 & \multirow{2}{*}{$\begin{array}{l}\text { Searched in the field for "Title" } \\
\text { Searched in the field for "Title" }\end{array}$} \\
\hline & ti:remedia* ti:interpersonal* & 43 & \\
\hline \multicolumn{2}{|c|}{ Total numbers of references } & & 614 \\
\hline \multicolumn{2}{|l|}{ Duplicates removed } & & 207 \\
\hline \multicolumn{2}{|c|}{ Total numbers of references after de-duplication } & & 407 \\
\hline
\end{tabular}

\section{Abbreviations}

ACGME: Accreditation Council for Graduate Medical Education; ABMS: American Board of Medical Specialties; BOE-PG: Board of Examiners for Postgraduate Programs; CCS: Communication and Consultation Skills; CPX: Clinical Performance Examination; CE: Certifying Exam; CME: Continuing Medical Education; CS: Clinical Skills; ECFMG: Educational Commission for Foreign Medical Graduates; FSMB: Federation of State Medical Boards; IEP: Individualized Education Plan; IRRC: Internal Residency Review Committee; MERSQI: Medical Education Research Study Quality Instrument; NBME: National Board of Medical Examiner; OSCE: Objective Structured Clinical Examination; PD: Program Director; PDI: Patient-Doctor Interaction; PIC: Professional Inspection Committee; SOI: Structured Oral Interview; SP: Standardized Patient: SPE: Standardized Patient Educator

\section{Acknowledgements}

The authors would like to acknowledge the support of the Johns Hopkins University Master of Education in the Health Professions (MEHP) Program, Toni Ungaretti, PhD and Sarah L. Clever, MD.

\section{Declaration of interest}

This systematic review was performed as part of a thesis for Master of Education in the Health Professions (MEHP) Program, Johns Hopkins University, USA. All authors report no declarations of interest.

\section{Authors' contributions}

LO created the search strategy and conducted the literature search. DA and TA screened the articles using the Covidence software. DA extracted the data, evaluated the quality of the studies and was the major contributor in writing the manuscript. The authors read and approved the final manuscript.

\section{Funding}

The publication of this article was funded by the Qatar National Library. Open Access funding provided by the Qatar National Library.

\section{Availability of data and materials}

All data generated or analysed during this study are included in this published article.

\section{Ethics approval and consent to participate}

Not applicable.

\section{Consent for publication}

Not applicable.

\section{Competing interests}

The authors declare that they have no competing interests.

\section{Author details}

'Division of Continuing Professional Development, Weill Cornell Medicine Qatar, Doha, Qatar. ${ }^{2}$ National Medical Library, United Arab Emirates
University, Al-Ain, UAE. ${ }^{3}$ Medical Education and Continuing Professional Development, Weill Cornell Medicine - Qatar, Doha, Qatar.

Received: 26 September 2019 Accepted: 12 May 2020

Published online: 09 July 2020

References

1. Batalden P, Leach D, Swing S, Dreyfus H, Dreyfus S. General competencies and accreditation in graduate medical education. Health Aff. 2002;21(5):103-11.

2. Epstein RM, Franks P, Fiscella K, Shields CG, Meldrum SC, Kravitz RL, et al. Measuring patient-centered communication in patient-physician consultations: theoretical and practical issues. Soc Sci Med. 2005;61(7):1516-28.

3. Epstein RM, Hundert EM. Defining and assessing professional competence. Jama. 2002;287(2):226-35.

4. Horowitz SD. Evaluation of clinical competencies: basic certification subspecialty certification, and recertification. Am J Phys Med Rehabil. 2000 79(5):478-80.

5. Makoul G. Communication skills education in medical school and beyond. Jama. 2003;289(1):93

6. Browning DM, Solomon MZ. Relational learning in pediatric palliative care: transformative education and the culture of medicine. Child Adolesc Psychiatr Clin N Am. 2006;15(3):795-815.

7. Contro N, Larson J, Scofield S, Sourkes B, Cohen H. Family perspectives on the quality of pediatric palliative care. Arch Pediatr Adolesc Med. 2002; 156(1):14-9

8. Macdonald ME, Liben S, Carnevale FA, Rennick JE, Wolf SL, Meloche D, et al. Parental perspectives on hospital staff members' acts of kindness and commemoration after a child's death. Pediatrics. 2005;116(4):884-90.

9. Meert KL, Thurston CS, Thomas R. Parental coping and bereavement outcome after the death of a child in the pediatric intensive care unit. Pediatr Crit Care Med. 2001;2(4):324-8.

10. Meyer EC, Ritholz MD, Burns JP, Truog RD. Improving the quality of end-oflife care in the pediatric intensive care unit: parents' priorities and recommendations. Pediatrics. 2006;117(3):649-57.

11. Azoulay E, Chevret S, Leleu G, Pochard F, Barboteu M, Adrie C, et al. Half the families of intensive care unit patients experience inadequate communication with physicians. Crit Care Med. 2000;28(8):3044-9.

12. Davies B, Connaughty S. Pediatric end-of-life care: lessons learned from parents. J Nurs Adm. 2002:32(1):5-6.

13. Kirchhoff KT, Walker L, Hutton A, Spuhler V, Cole BV, Clemmer T. The vortex: families' experiences with death in the intensive care unit. Am J Crit Care. 2002;11(3):200-9.

14. Anderson M, Cohen J, Hallock J, Kassebaum D, Turnbull J, Whitcomb M. Learning objectives for medical student education-guidelines for medical schools: report I of the medical school objectives project. Acad Med. 1999;74(1):13-8.

15. Colleges AAM. Learning objectives for medical student education: guidelines for medical schools: association; 1998

16. Cowan D, Danoff D, Davis A, Degner L, Jerry M, Kurtz S, et al. Consensus statement from the workshop on the teaching and assessment of communication skills in Canadian medical schools. Can Med Assoc J. 1992 147(8):1149-50 
17. Makoul G, Schofield T. Communication teaching and assessment in medical education: an international consensus statement. Patient Educ Couns. 1999: 37(2):191-5

18. United States Medical Licensing Examination. [Available from: http://www. usmle.org/frequently-asked-questions/\#step2cs. Accessed 22 Jan 2018.

19. Sullivan C, Murano T, Comes J, Smith JL, Katz ED. Emergency medicine directors' perceptions on professionalism: a council of emergency medicine residency directors survey. Acad Emerg Med. 2011;18(10 SUPPL. 2):S97-S103.

20. Torbeck L, Canal DF. Remediation practices for surgery residents. Am J Surg. 2009;197(3):397-402.

21. Regan L, Hexom B, Nazario S, Chinai SA, Visconti A, Sullivan C. Remediation methods for milestones related to interpersonal and communication skills and professionalism. J Grad Med Educ. 2016;8(1):18-23.

22. Katz ED, Dahms R, Sadosty AT, Stahmer SA, Goyal D. Guiding principles for resident remediation: recommendations of the CORD remediation task force. Acad Emerg Med. 2010;17(s2):S95-S103.

23. Hauer KE, Ciccone A, Henzel TR, Katsufrakis P, Miller SH, Norcross WA, et al. Remediation of the deficiencies of physicians across the continuum from medical school to practice: a thematic review of the literature. Acad Med. 2009;84(12):1822-32

24. Saxena V, O'Sullivan PS, Teherani A, Irby DM, Hauer KE. Remediation techniques for student performance problems after a comprehensive clinical skills assessment. Acad Med. 2009;84(5):669-76.

25. White CB, Ross PT, Gruppen LD. Remediating students' failed OSCE performances at one school: the effects of self-assessment, reflection, and feedback. Acad Med. 2009;84(5):651-4.

26. Hauer KE, Teherani A, Irby DM, Kerr KM, O'Sullivan PS. Approaches to medical student remediation after a comprehensive clinical skills examination. Med Educ. 2008;42(1):104-12.

27. Frellsen SL, Baker EA, Papp KK, Durning SJ. Medical school policies regarding struggling medical students during the internal medicine clerkships: results of a national survey. Acad Med. 2008;83(9):876-81.

28. Chou CL, Bell J, Chou CM, Chang A. Remediation of interpersonal and communication skills. In: Remediation in medical education. New York: Springer; 2014. p. 55-66.

29. Guerrasio J, Furfari KA, Rosenthal LD, Nogar CL, Wray KW, Aagaard EM. Failure to fail: the institutional perspective. Med Teach. 2014;36(9):799-803.

30. Moher D, Liberati A, Tetzlaff J, Altman DG, The PRISMA Group. Preferred Reporting Items for Systematic Reviews and Meta-Analyses: The PRISMA Statement. PLoS Med. 2009;6(7):e1000097. https://doi.org/10.1371/journal. pmed1000097.

31. Reed DA, Cook DA, Beckman TJ, Levine RB, Kern DE, Wright SM. Association between funding and quality of published medical education research. Jama. 2007;298(9):1002-9.

32. Kirkpatrick DL. The four levels of evaluation. In: Evaluating corporate training: models and issues. Switzerland: Springer; 1998. p. 95-112.

33. Deveugele M, Derese A, Maesschalck SD, Willems S, Driel MV, Maeseneer JD. Teaching communication skills to medical students, a challenge in the curriculum? Patient Educ Couns. 2005;58(3):265-70.

34. Dowell J, Dent JA, Duffy R. What to do about medical students with unsatisfactory consultation skills? Med Teach. 2006;28(5):443-6.

35. Lin CT, Barley GE, Cifuentes M. Personalized remedial intensive training of one medical student in communication and interview skills. Teach Learn Med. 2001:13(4):232-9.

36. Rowland PA, Trus TL, Lang NP, Henriques H, Reed WP Jr, Sadighi PJ, et al. The certifying examination of the american board of surgery: the effect of improving communication and professional competency: twenty-year results. J Surg Educ. 2012:69(1):118-25.

37. Ryan CA, Walshe N, Gaffney R, Shanks A, Burgoyne L, Wiskin CM. Using standardized patients to assess communication skills in medical and nursing students. BMC Med Educ. 2010;10:24. https://doi.org/10.1186/ 1472-6920-10-24.

38. Sperry JA, Williams D, Guiffre AM. Addressing communication skill deficits in fourth-year medical students: an intensive remediation curriculum. Ann Behavioral Sci Med Educ. 2010;16(1):14-20.

39. Wiskin C, Doherty EM, Von Fragstein M, Laidlaw A, Salisbury H. How do United Kingdom (UK) medical schools identify and support undergraduate medical students who 'fail' communication assessments? A national survey. BMC Med Educ. 2013:13:95. https://doi.org/10.1186/1472-6920-13-95.
40. Zbieranowski I, Takahashi SG, Verma S, Spadafora SM. Remediation of residents in difficulty: a retrospective 10-year review of the experience of a postgraduate board of examiners. Acad Med. 2013;88(1):111-6.

41. Chang A, Chou CL, Hauer KE. Clinical skills remedial training for medical students. Med Educ. 2008;42(11):1118-9.

42. Goulet F, Jacques A, Gagnon R. An innovative approach to remedial continuing medical education, 1992-2002. Acad Med. 2005;80(6):533-40.

43. Myung SJ, Yim J-J, Park SM, Shin JS. Clinical-performance remediation program for dyscompetent medical students. Kor J Med Educ. 2013;25(2):123-9.

44. Saxena V. OSPSTAIDMHKE. Remediation techniques for student performance problems after a comprehensive clinical skills assessment. Acad Med. 2009; 84(5):669-76.

45. Bodenberg MM, Koehler JM. Customizing student learning during advanced pharmacy practice experiences. Curr Pharm Teach Learn. 2015;7(4):519-25.

46. Guevara M, Grewald Y, Hutchinson K, Amoateng-Adjepong Y, Manthous C. Individualized education plans in medical education. Conn Med. 2011;75(9):537-40.

47. Malau-Aduli BS, Page W, Cooling N, Turner R. Impact of self-efficacy beliefs on short-and long-term academic improvements for underperforming medical students. Am J Educ Res. 2013;1(6):168-76.

48. Duffy FD, Gordon GH, Whelan G, Cole-Kelly K, Frankel R. Assessing competence in communication and interpersonal skills: the Kalamazoo II report. Acad Med. 2004;79(6):495-507.

49. Hodges B, Regehr G, Hanson M, McNaughton N. An objective structured clinical examination for evaluating psychiatric clinical clerks. Acad Med. 1997;72(8):715-21.

50. Miller GE. The assessment of clinical skills/competence/performance. Acad Med. 1990:65(9):S63-7.

51. Schirmer JM, Mauksch L, Lang F, Marvel MK, Zoppi K, Epstein RM, et al. Assessing communication competence: a review of current tools. Fam Med. 2005;37(3):184-92.

52. Hauer KE, Teherani A, Kerr KM, O'Sullivan PS, Irby DM. Student performance problems in medical school clinical skills assessments. Acad Med. 2007; 82(10):S69-72

53. Makhani L, Bradley R, Wong J, Krynski E, Jarvis A, Szumacher E. A framework for successful remediation within allied health programs: strategies based on existing literature. J Med Imaging Rad Sci. 2012:43(2):112-20.

54. Hawkins R, Roemheld-Hamm B, Ciccone A, Mee J, Tallia A. A multimethod study of needs for physician assessment: implications for education and regulation. J Contin Educ Health Prof. 2009;29(4):220-34.

55. Cleland J, Leggett H, Sandars J, Costa MJ, Patel R, Moffat M. The remediation challenge: theoretical and methodological insights from a systematic review. Med Educ. 2013;47(3):242-51.

56. Boiselle PM. A remedy for resident evaluation and remediation 1. Acad Radiol. 2005;12(7):894-900.

57. Kosir MA, Fuller L, Tyburski J, Berant L, Yu M. The Kolb learning cycle in American Board of Surgery in-training exam remediation: the accelerated clinical education in surgery course. Am J Surg. 2008;196(5):657-62.

58. Kolb D. Experiential education: experience as the source of learning and development. Englewood Cliffs: Prentice Hall; 1984.

59. Leung F-H, Martin D, Batty H. A theory-based curriculum design for remediation of residents' communication skills. Med Teach. 2009:31(12):e555-e9.

60. Bloom BS. Taxonomy of educational objectives: the classification of educational goals: cognitive domain. London: Longman; 1956.

61. Bandura A. Social foundations of thought and action: a social cognitive theory. Englewood Cliffs: Prentice-Hall, Inc; 1986

62. Schon D. Educating the reflective practitioner. San Francisco: Jossey-Bass; 1987.

63. Hatem D. The reflection competency: using narrative in remediation. In: Remediation in medical education. New York: Springer; 2014. p. 235-48.

64. Borus JF. Recognizing and managing residents' problems and problem residents. Acad Radiol. 1997:4(7):527-33.

\section{Publisher's Note}

Springer Nature remains neutral with regard to jurisdictional claims in published maps and institutional affiliations. 